\title{
Helicteres L. SPECIES (Malvaceae SENSU LATO) AS SOURCE OF NEW DRUGS: A REVIEW
}

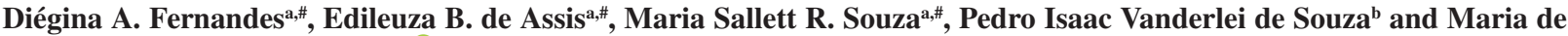

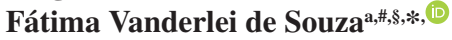

a'Departamento de Ciências Farmacêuticas, Universidade Federal da Paraíba, 58051-900 João Pessoa - PB, Brasil

bInstituto de Biociências, Universidade Federal do Mato Grosso do Sul, 79070-900 Campo Grande - MS, Brasil

Recebido em 11/11/2019; aceito em 12/02/2020; publicado na web em 20/04/2020

\begin{abstract}
Helicteres genus, Malvaceae, has pantropical distribution, encompasses about 60 species, 31 of them found in Brazil. Species belonging to this genus are used for treatment of various diseases and aroused scientific interest in search for bioactive compounds present in these plants. In this context, this review aims to provide a complete and concise overview of scientific advances in phytochemical and pharmacological studies of these species and their use by folk medicine. The presented data were collected from scientific databases, 'Web of Science', 'Scifinder', 'Pubmed', 'Sciencedirect', and 'Google Scholar', using the keyword 'Helicteres'. The species H. isora and H. angustifolia, found in Asia, are the most explored scientifically, whereas studies of species of this genus found in Americas are still rare, being possible to highlight studies carried out in Brazil with $H$. velutina and H. eichleri. About 149 compounds were isolated and characterized in the genus, being emphasized terpenoids, flavonoids and lignoids. These species have demonstrated various pharmacological properties in vitro and in vivo, incluinding insecticide, antidiabetic, antitumor and hepatoprotective activities. The presented data show the importance of studies carried out isolating bioactive compounds from this genus that may be used in several diseases' treatment or/as prototypes to development of new drugs.
\end{abstract}

Keywords: Helicteres L.; secondary metabolite; ethnopharmacological relevance; scientific studies.

\section{INTRODUCTION}

The use of natural products by mankind with the purpose of supplying physical and biological needs is an ancient practice, being the knowledge acquired transmitted throughout the generations. ${ }^{1-3}$ Previous studies have allowed the association of chemical constituents present in the medicinal species and their respective pharmacological activities, based on experimental researches including knowledges of botany, chemistry, biochemistry and pharmacology, greatly contributing to the discovery of bioactive natural products. ${ }^{4,5}$

In this context, species of Sterculiaceae clade, Malvaceae sensu lato, have aroused great interest in the scientific environment and stand out for their importance in industrial, economic, medicinal, food and ornamental production, as well their chemical and biological properties. $^{6,7}$

Among the genus belonging to this group, we highlight Helicteres L., whose biological and pharmacological effects of some species used in folk medicine were scientifically confirmed through the isolation, structural characterization and pharmacological activities developed by its secondary metabolites. ${ }^{8}$

Helicteres L. genus has pantropical distribuition, comprising approximately 60 species in America and Asia, with no species common to both continents. In Asia, the most studied species chemically and pharmacologically are $H$. isora, $H$. angustifolia and $H$. hirsute. China has about ten species, of which only one is endemic. ${ }^{9}$

In America, there are 38 species distributed from Mexico to Argentina, with no reports of occurence for Ecuador and Chile. Among the most scientifically studied species in the continent, we can highlight $H$. sacarolha, $H$. eichleri and $H$. velutina, the last two endemic in Brazil, which is considered the center of diversity of this

*e-mail: mfvanderlei@1tf.ufpb.br

\#Programa de Pós-graduação em Produtos Naturais e Sintéticos Bioativos

${ }^{\S}$ Programa de Pós-graduação em Desenvolvimento e Inovação Tecnológica em Medicamentos genus in Americas, having a registered occurrence of 31 species, 23 of which are exclusive from cerrado, caatinga and dry forests. ${ }^{10-12}$

Based on the presented data, the objective of this review is to make a survey about the traditional use of Helicteres genus species, as well as evaluating their chemical and pharmacological potential to show the importance of this genus and provide a basis for future research.

\section{METHODOLOGY}

Available information on traditional uses, phytochemical study, botanical characteristics and biological activities of Helicteres genus were collected from scientific databases: 'Web of Science', 'Scifinder', 'Pubmed', 'Sciencedirect', and 'Google Scholar', using articles, books, dissertations and theses published until July 2019, using the keyword 'Helicteres'. This way, we came across 173 scientific papers. The interest in scientific studies focusing on species of this genus for the development of new drugs has increased over the years due to the promising results of the scientific studies conducted with Helicteres.

The study selection and data extraction were performed by one author (DAF) and confirmed by others (EBA, MSRS, MFVS). The extracted data were summarized in tabular form and a narrative description was used to provide a summary of updated information.

\section{RESULTS AND DISCUSSION}

\section{Botanical profile and pollination}

The Helicteres L. species are characterized morphologically as erect shrubs or sub-bushes, with showy, zygomorph, pedicellate flowers usually pendulous and odorless (Figure 1), with a long androgynophore and ten transverse stamens grafted in to a yellow to red corolla; fruits are distinct, spiral capsules. ${ }^{13-15}$

Its showy flowers are strongly attracted to bats and hummingbirds, as described in studies with $H$. ovata ${ }^{16} \mathrm{H}$. brevispira, $\mathrm{H}$. sacarolha, ${ }^{17-19}$ and $H$. lhotzkyana. ${ }^{14,20} H$. isora flowers, present at Asia, are large and 

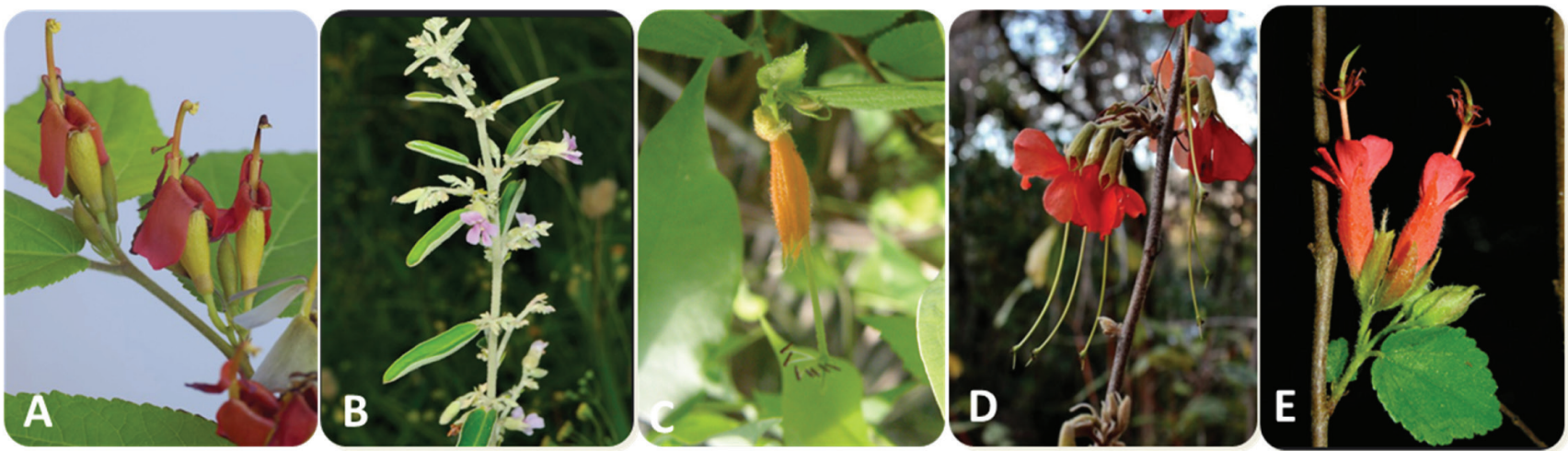

Figure 1. Helicteres plants. A) H. isora, B) H. angustifolia, C) H. sacarolha, D) H. brevispera, E) H. grazumifolia ${ }^{15}$

open daily, being visited by birds and insects that assist in pollination during the day. ${ }^{21}$

\section{Ethnopharmacological relevance}

Almost all parts of Helicteres L. plants, including roots, bark and aerial parts, are reported to be traditionally used in several countries and tribes for therapeutic purposes (Table 1).

The juice of $H$. isora root is used in Tradicional Chinese Medicine for diabetes treatment, while fruit extract is used in various intestinal disorders to relieve colic and as an anthelmintic medicine against tapeworm. ${ }^{22-24}$

The root tea of $H$. angustifolia is used to treat influenza symptoms and to inhibit tumor growth. ${ }^{25} \mathrm{H}$. sacarolha preparations with roots and leaves, in form of decoction, infusion or maceration, are used for liver complications, ovarian inflammation, amenorrhea and blood purification, ${ }^{8}$ while the aerial parts of $H$. velutina are used by indigenous tribe Pankarare/Brazil as insect repellent. ${ }^{26}$

Ethnopharmacological research with species of this genus acts as a subsidy to the pharmaceutical interest and registration of the empirical uses of medicinal plants in traditional communities associated with chemical-pharmacological tests generates useful knowledges to lead to the development of new drugs.

\section{Phytochemistry profile}

In literature, 46 references on the phytochemistry field with species of Helicteres genus were found, 39 of which referring to the studies with $H$. isora and $H$. angustifolia. Furthemore, papers reporting research in this area with the species $H$. hirsute, ${ }^{38,46}$ $H$. vegae ${ }^{47} H$. velutina ${ }^{12}$ and $H$. eichler ${ }^{48}$ were found, 149 compounds were isolated and identified from Helicteres (Table 2) among the most reported classes. All substances are compiled in the Table 2 (compounds) and Figure 2 (structures).

\section{Terpenoids and steroids}

Terpenoids and its oxygenated, acetylated and dehydrogenated derivatives are hydrocarbons of plant origin. ${ }^{84}$ Many of these molecules have biological activities that are used for the treatment of human diseases. These molecules have led to six major classes of drugs in the last century: steroids, tocopherols, texanes, artemisinins, ingenans and cannabinoids. ${ }^{85,86}$

Fifty terpenoids were isolated and identified from $H$. isora, $H$. angustifolia, $H$. hirsuta, $H$. eichleri and $H$. velutina, evidencing this class as the predominant compounds in Helicteres genus (Table 2). In a preliminary bioassay, cucurbitacins $\mathrm{D}(\mathbf{4})$ and $\mathbf{J}(\mathbf{8})$ exhibited significant inhibitory activities against hepatocellular carcinoma and malignant melanoma cells in vitro. ${ }^{51}$
Compounds $3 \beta-O$ (trans-coumaroyl) betulinic acid (15), pyracrenic acid (16), 3 $\beta$-acetoxy-27-[(4-hydroxybenzoyl)oxy]lup20(29)-en-28-oic acid (32) and 3 $\beta$-acetoxy-27-[(4-hydroxybenzoyl) oxy]olean-12-en-28-oic acid methyl (39), showed significant cytotoxic activities against human colorectal cancer and human gastric cancer cell lines in vitro. ${ }^{44}$ The compound 10-methyl, 4-isopropenyl, dodecahydro-ethanophenanthrene (48) exhibited considerable antimicrobial and antispasmodic activities. ${ }^{64}$

Steroids are one of the less widespread classes in isolated from species of Helicteres genus, with only seven representatives (50-56).

Phytosteroids are steroidal substances extracted from plant species, the most common being $\beta$-sitosterol (51) and Stigmasterol (52). This class of substances has carbonic skeleton formed by the cyclopentanoperhydrophenanthrenic ring, ${ }^{87}$ highlightin the $\beta$-sitosterol, which presented antimicrobial and larvicidal activities. $^{88,89}$

\section{Flavonoids and phenolic compounds}

Flavonoids represent one of the most important and diverse groups of phenolic compounds among natural products. ${ }^{90,91}$ Among the phytotherapics currently studied, flavonoids have been highlighted due to their wide range of biological and/or pharmacological actions demonstrated under both experimental and human conditions. ${ }^{92}$

Twenty-nine flavonoids were isolated from Helicteres genus, with emphasis on heterosides (69), ${ }^{36,71}$ sulphated (78-80) and heterosides glycosulphated (65-67). ${ }^{12,36}$ Among those compounds, tiliroside (70) and 7,4'-di- $O$-methyl-8- $O$-sulphate flavone (78) have larvicidal activity against Aedes aegypti, ${ }^{88}$ while $7,4^{\prime}$ - $O$-methylisoscutellarein (60) have shown anti-inflammatory activity by inhibiting neutrophil recruitment and decreasing IL- $1 \beta$ and TNF- $\alpha$ production in vitro. ${ }^{93}$

Besides the flavonoids, it was possible to identify 18 phenolic compounds (85-102) with different nuclei, among them rosmarinic acid (85) isolated from $H$. isora fruits, $H$. angustifolia and $H$. vegae roots and $H$. eichleri aerial parts. Scientific studies of this substance have proven its antioxidant, anti-inflammatory, antifibrosis, hepatoprotective and antineoplasic activities. ${ }^{94}$

Some studies also report the compounds quantification in species of Helicteres genus, among which are total phenolic content, flavonoids and condensed tannins of $H$. vegae. ${ }^{47}$ It was also accomplished the phenolics, flavonoids and saponins quantification of $H$. hirsuta ${ }^{45,95,96}$ and $H$. isora, to evaluate their antioxidant potential. ${ }^{97-99}$

\section{Lignoids}

Twenty-one lignoids were isolated and identified from $H$. isora, $H$. angustifolia, $H$. hirsuta and $H$. velutina species, most of them found in the roots and fruits. Yin et al. (2016) ${ }^{57}$ isolated two benzofuran lignans of $H$. angustifolia that were evaluated for anti-complementary activity in vitro and showed potent activity when compared to heparin 
Table 1. Species of Helicteres genus and their uses in folk medicine

\begin{tabular}{|c|c|c|c|c|}
\hline $\begin{array}{l}\text { Scientific name/ } \\
\text { Popular name }\end{array}$ & Medicinal parts & Traditional use & Therapeutic indications & References \\
\hline \multirow[t]{3}{*}{ H. isora / "Ulet-Ulet" } & RT and BK & $\begin{array}{l}\text { Decoction } \\
\text { Juice } \\
\text { Paste } \\
\text { Extract }\end{array}$ & $\begin{array}{l}\text { Anthelmintic } \\
\text { Snake bites } \\
\text { Chronic nephritis } \\
\text { Gastric ulcers } \\
\text { Antidiabetic } \\
\text { Expectorant } \\
\text { Astringent } \\
\text { Anticolagogue } \\
\text { Antiasthmatic } \\
\text { Intestinal infections }\end{array}$ & $22-24,27-34$ \\
\hline & FR & Pod extracts & $\begin{array}{c}\text { Anthelmintic } \\
\text { Intestinal infections } \\
\text { Vermifuges } \\
\text { Antiflatulence } \\
\text { Antispasmodic } \\
\text { Astringent } \\
\text { Antidiarrheal } \\
\text { Antidysenteric } \\
\text { Antipyretic }\end{array}$ & $22,24,27,29-32,34-38$ \\
\hline & LV & Paste & Tanning & 30,39 \\
\hline $\begin{array}{l}\text { H. angustifolia / } \\
\text { "Shan-Zhi-Ma" }\end{array}$ & RT and ST & $\begin{array}{c}\text { Tea } \\
\text { Medicinal liquor }\end{array}$ & $\begin{array}{c}\text { Analgesic } \\
\text { Anti-inflammatory } \\
\text { Antibacterial } \\
\text { Antiviral } \\
\text { Antitumoral }\end{array}$ & $25,40-42$ \\
\hline H. sacarolha / "Sacarolha" & RT and LV & $\begin{array}{l}\text { Decoction } \\
\text { Infusion } \\
\text { Maceration }\end{array}$ & $\begin{array}{l}\text { Antihypertensive } \\
\text { Antiulcerogenic } \\
\text { Antisyphilitic } \\
\text { Hepatoprotective } \\
\text { Anti-inflammatory } \\
\text { Amenorrhea } \\
\text { Blood clearance }\end{array}$ & $8,25,43$ \\
\hline H. velutina /"Pitó" & $\mathrm{AP}$ & $*$ & Insect repellent & 26 \\
\hline
\end{tabular}

*not reported in the literature. RT: Roots; BK: Barks; FR: Fruits; LV: Leaves; ST: Stems; AP: Aerial Parts.

(positive control). Tezuka et al. $(2000)^{74}$ isolated and identified six dimeric neolignans from fruits of $H$. isora: Helicterins B (120), $\mathrm{C}$ (115), D (117), E (116) and F (118), which showed mild inhibitory activity against avian myeloblastosis virus reverse transcriptase (AMV-RT), having an emphasis on the inhibitory activity of Helicterins A (119), which was identical to the antineoplasic drug doxirubicin, with an $\mathrm{IC}_{50}$ of $66 \mu \mathrm{M}$. This can be of interest for the development of new therapeutic alternatives.

\section{Quinones}

Quinones are structurally characterized as cyclic $\alpha, \beta$-dienics, and have considerable toxicological and pharmacological interests due to their biooxidation-reduction properties and ability to catalyze biological electrical transfer. ${ }^{100}$ However, biological studies involving isolated quinones of Helicteres species are scarce, as it is necessary to investigate possible biological actions not yet explored. So far, ten quinones have been isolated and identified in the studied genus (123-132), and the compounds that best represent this class were sesquiterpene quinones and $O$-benzoquinones, isolated from H. angustifolia roots. ${ }^{40,42,51}$
Other compounds

Beyond to previously detailed compounds, other classes of metabolites, such as amines, saponins, lactones, coumarins, alcohols, fatty acids, alkaloids, pheophytins and tannins (133-149) (Table 2, Figure 1), were less frequently detected in this genus.

Aleykutty \& Akhila (2012), ${ }^{80}$ by means of a computational approach, predicted the antidiabetic potential of the chemical constituents identified in $H$. isora, especially the indolalkylamine, Yohimbine (142), which presented the best binding energy with the enzyme aldose reductase and the insulin receptor protein, pharmacological targets for glycemic control.

\section{Pharmacology study}

Pharmacological potential of Helicteres species has gained prominence, especially with $H$. isora and $H$. angustifolia, that have a long history of use in traditional Chinese medicine. Researches have been developed about antidiabetic, antiulcerogenic and antitumor activities within Helicteres species in order to confirm the activities reported by folk medicine (Table 3 ). 
Table 2. Isolated compounds from Helicteres genus

\begin{tabular}{|c|c|c|c|}
\hline $\mathrm{N}^{\circ}$ & Name & Source & Literature \\
\hline \multicolumn{4}{|c|}{ Terpenoids } \\
\hline 1 & Cucurbitacin B & RT of $\boldsymbol{H} . \boldsymbol{i}$. & 24,49 \\
\hline 2 & Cucurbitacin B 2-sulfate & RT of $\boldsymbol{H} . \boldsymbol{a}$. & 50 \\
\hline 3 & Isocucurbitacin B & RT of $\boldsymbol{H} . \boldsymbol{i}$. & 24,49 \\
\hline 4 & Cucurbitacin D & \multirow{7}{*}{ RT of $\boldsymbol{H} . \boldsymbol{a}}$. & \multirow{7}{*}{$50-53$} \\
\hline 5 & Cucurbitacin E & & \\
\hline 6 & Cucurbitacin G 2-O- $\beta$-D-glucopyranoside & & \\
\hline 7 & Cucurbitacin I & & \\
\hline 8 & Cucurbitacin J & & \\
\hline 9 & Isocurcubitacin D & & \\
\hline 10 & Hexanorcucurbitacin I & & \\
\hline 11 & Lup-20(29)-em-3 $\beta$-ol & $\begin{array}{l}\text { RT of } \boldsymbol{H} . \boldsymbol{h} . \\
\text { AP of } \boldsymbol{H} . \boldsymbol{e} .\end{array}$ & 48,54 \\
\hline 12 & Betulinic acid & RT of $\boldsymbol{H . i . , H}$.a. and $\boldsymbol{H} . \boldsymbol{h}$. & $51,54,55$ \\
\hline 13 & $3 \beta$-benzoylbetulinic acid & \multirow{2}{*}{ RT of $\boldsymbol{H} . \boldsymbol{h}}$. & \multirow{2}{*}{54} \\
\hline 14 & Betulinic acid methyl ester & & \\
\hline 15 & $3 \beta-O$ (trans-coumaroyl) betulinic acid & RT of $\boldsymbol{H} . \boldsymbol{a}$. & 44 \\
\hline 16 & Pyracrenic acid & RT of $\boldsymbol{H} . \boldsymbol{a}$. and $\boldsymbol{H} . \boldsymbol{h}$. & 53 \\
\hline 17 & $3 \beta-O$-(trans-feruloyl) betulinic acid & \multirow{5}{*}{ RT of $\boldsymbol{H} . \boldsymbol{a}}$. & \multirow{5}{*}{44} \\
\hline 18 & $3 \beta-O$-(trans-coumaroyl) botulin & & \\
\hline 19 & $3 \beta-O$-( cis-coumaroyl) botulin & & \\
\hline 20 & $3 \beta-O$-(trans-caffeoyl) betulin & & \\
\hline 21 & $3 \beta-O$-(trans-feruloyl) betulin & & \\
\hline 22 & $3 \beta$-acetoxybetulinic acid & $\begin{array}{l}\text { RT of } \boldsymbol{H} . \boldsymbol{a} . \\
\text { AP of } \boldsymbol{H} . \boldsymbol{h} .\end{array}$ & 52,56 \\
\hline 23 & 3-acetoxybetulin & \multirow{11}{*}{ RT of $\boldsymbol{H . a .}$} & \multirow{11}{*}{$35,44,51,53,55$} \\
\hline 24 & 3ß-27-diacetoxy-lup-20(29)en-28-oic methyl ester & & \\
\hline 25 & $3 \beta$-acetoxy-27-benzoyloxylup-20(29)-en-28-oic acid & & \\
\hline 26 & $3 \beta$-acetoxylup-20(29)-en-28-ol & & \\
\hline 27 & 3ß-hydroxylup-20(29)-en-28-oic acid 3-caffeate & & \\
\hline 28 & 3ß-hydroxy-27-benzoyloxylup-20(29)-en-28-oic acid & & \\
\hline 29 & 3ß-hydroxy-27-benzoyloxylup-20(29)-en-28-oic acid methyl ester & & \\
\hline 30 & Methyl helicterate & & \\
\hline 31 & $3 \beta$-acetoxy-27-[(E)-cinnamoyloxy $]$ lup-20(29)-en-28-oic acid methyl ester & & \\
\hline 32 & 3ß-acetoxy-27-[(4-hydroxybenzoyl)oxy]lup-20(29)-en-28-oic acid & & \\
\hline 33 & Cylicodiscic acid & & \\
\hline 34 & Simiarenol & AP of $\boldsymbol{H} . \boldsymbol{h}$. & 56 \\
\hline 35 & Isorin & RT and FR of $\boldsymbol{H} . \boldsymbol{i}$. & 55,57 \\
\hline 36 & 3ß-hydroxyolean-12-en-27-benzoyloxy-28-oate & \multirow{5}{*}{ RT of $\boldsymbol{H . a}$. } & \multirow{5}{*}{$44,58-60$} \\
\hline 37 & $3 \beta-O$-( $p$-hydroxy-(E)-cinnamoyl)-12 oleanen-28-oic acid & & \\
\hline 38 & Helicterilic acid & & \\
\hline 39 & $3 \beta$-acetoxy-27-[(4-hydroxybenzoyl)oxy]olean-12-en-28-oic acid methyl ester & & \\
\hline 40 & $3 \beta$-acetoxy-27-(benzoyloxy)olean-12-en-28-oic acid methyl ester & & \\
\hline 41 & Ursolic acid & $\begin{array}{l}\text { RT of } \boldsymbol{H} . \boldsymbol{a} . \\
\text { AP of } \boldsymbol{H} . \boldsymbol{e} .\end{array}$ & 48,61 \\
\hline$\overline{42}$ & $3 \alpha$-hydroxy-urs-12-en-28-oic acid & \multirow{3}{*}{ AP of $\boldsymbol{H . e .}$} & \multirow{3}{*}{48} \\
\hline 43 & $3 \alpha$-hydroxy-olean-12-en-28-oic acid & & \\
\hline 44 & Micromeric acid & & \\
\hline 45 & Oleanolic acid & $\begin{array}{l}\text { RT of } \boldsymbol{H} . \boldsymbol{i} ., \boldsymbol{H} . \boldsymbol{a} . \\
\text { AP of } \boldsymbol{H . v e l .}\end{array}$ & $12,62,63$ \\
\hline 46 & $3 \beta$-acethoxy-olean-12-en-28-oic acid & \multirow{2}{*}{ AP of $\boldsymbol{H} . v e l$. } & \multirow{2}{*}{12} \\
\hline 47 & $3 \beta$-sterearyloxy-olean-12-ene & & \\
\hline 48 & 10-methyl, 4-isopropenyl, dodecahydro-ethanophenanthrene & RT of $\boldsymbol{H} . \boldsymbol{i}$. & 64 \\
\hline 49 & Methyl helicterilate & RT of $\boldsymbol{H} . \boldsymbol{a}$. & 65 \\
\hline
\end{tabular}


Table 2. Isolated compounds from Helicteres genus (cont.)

\begin{tabular}{|c|c|c|c|}
\hline $\mathrm{N}^{\mathrm{o}}$ & Name & Source & Literature \\
\hline \multicolumn{4}{|c|}{ Steroids } \\
\hline 50 & $\beta$-sitosterol glucoside & $\begin{array}{c}\text { RT of } \boldsymbol{H} . \boldsymbol{i} ., \boldsymbol{H} . \boldsymbol{a} . \\
\text { AP of } \boldsymbol{H} . \boldsymbol{v e l} .\end{array}$ & $12,24,66$ \\
\hline 51 & $\beta$-sitosterol & $\begin{array}{c}\text { RT of } \boldsymbol{H} . \boldsymbol{i} ., \boldsymbol{H} . \boldsymbol{a} . \\
\text { AP of } \boldsymbol{H} . \boldsymbol{e} .\end{array}$ & $24,48,67,68$ \\
\hline 52 & Stigmasterol & $\begin{array}{l}\text { RT of } \boldsymbol{H} . \boldsymbol{h} . \\
\text { AP of } \boldsymbol{H} . \boldsymbol{e} .\end{array}$ & 48,54 \\
\hline 53 & Heligenin A & & \\
\hline 54 & Heligenin B & & \\
\hline 55 & 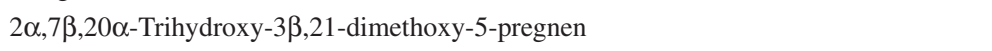 & RT of $\boldsymbol{H} \cdot \boldsymbol{a}$. & $51,52,66$ \\
\hline$\underline{56}$ & $3 \beta$-ergost-5-en-3-ol & & \\
\hline \multicolumn{4}{|c|}{ Flavonoids } \\
\hline 57 & Kaempferol-3-O-galactoside & & \multirow{2}{*}{69} \\
\hline$\underline{58}$ & Herbacetin-8-O-glucuronide & & \\
\hline 59 & 7-O-methylisoscutellarein & \multirow{3}{*}{ AP of $\boldsymbol{H} . \boldsymbol{h}$. } & \multirow{3}{*}{$54,56,70$} \\
\hline 60 & 7,4'-di- $O$-methylisoscutellarein & & \\
\hline 61 & Isoscutellarein 4'-methyl ether 8-O- $\beta$-D-glucopyranoside & & \\
\hline 62 & Isoscutellarein 4'-methyl ether 8-O- $\beta$-D-glucuronide 6"-n-butyl ester & \multirow{5}{*}{ FR of $\boldsymbol{H} . \boldsymbol{i}$. } & \multirow{5}{*}{36} \\
\hline 63 & Isoscutellarein 4'-methyl ether 8-O- $\beta$-D-glucuronide & & \\
\hline 64 & Isoscutellarein 4'-methyl ether 8-O- $\beta$-D-glucuronide 2 "'-sulfate & & \\
\hline 65 & Isoscutellarein 4'-methyl ether 8-O- $\beta$-D-glucuronide $2 ", 4 "$-disulfate & & \\
\hline 66 & Isoscutellarein 8-O- $\beta$-D-glucuronide 2",4"-disulfate & & \\
\hline 67 & Kaempferol-3-O- $\beta$-D-glucopyranoside & $\begin{array}{l}\text { FR of } \boldsymbol{H} \cdot \boldsymbol{i} . \\
\text { RT of } \boldsymbol{H} \cdot \boldsymbol{a} .\end{array}$ & $51,55,57$ \\
\hline 68 & Kaempferol & $\begin{array}{l}\text { FR of } \boldsymbol{H} . \boldsymbol{i} . \\
\text { AP of } \boldsymbol{H} . \boldsymbol{v} \boldsymbol{e l} .\end{array}$ & 12,57 \\
\hline 69 & Tiliroside & $\begin{array}{c}\text { FR of } \boldsymbol{H} . \boldsymbol{i} . \\
\text { RT of } \boldsymbol{H} \cdot \boldsymbol{a} . \\
\text { AP of } \boldsymbol{H . v e l .}\end{array}$ & $12,52,55,57$ \\
\hline 70 & 5,7,8-trihydroxy-4'-methoxyflavone & $\begin{array}{l}\text { FR of } \boldsymbol{H} . \boldsymbol{i} . \\
\text { AP of } \boldsymbol{H} . \boldsymbol{a} .\end{array}$ & 55,57 \\
\hline 71 & 3',5,7,8-tetrahydroxy-4'-methoxyflavone & FR of $\boldsymbol{H} . \boldsymbol{i}$. & 71 \\
\hline 72 & Takakin 8-O- $\beta$-D-glucuronide 6-methyl ester & \multirow{4}{*}{ RT of $\boldsymbol{H} . \boldsymbol{a}$. } & \multirow{4}{*}{72} \\
\hline 73 & Takakin 8-O- $\beta$-D-glucuronide 2-sodium sulfate & & \\
\hline 74 & Takakin 8-O- $\beta$-D-glucuronide & & \\
\hline 75 & 8-O- $\beta$-D-glucuronyl-hypolaetin 4'-methyl ether & & \\
\hline 76 & 5,8-dihydroxy-7,4'-dimethoxyflavone & $\begin{array}{c}\text { LV of } \boldsymbol{H} . \boldsymbol{i} . \\
\text { RT of } \boldsymbol{H} . \boldsymbol{a} . \\
\text { AP of } \boldsymbol{H . v e l .} \text { and } \boldsymbol{H . h .}\end{array}$ & $12,51,54,69$ \\
\hline 77 & Tricin & RT of $\boldsymbol{H} . \boldsymbol{a}$. & 66 \\
\hline 78 & 7,4'-di- $O$-metil-8-O-sulphate flavone & \multirow[t]{3}{*}{ AP of H.vel. } & \multirow[t]{3}{*}{12} \\
\hline 79 & 5,4'-di-hydroxy-7-methoxy-8-O-sulphate flavone & & \\
\hline 80 & 5,6-di-hydroxy-7,4'-methoxy-8-O-sulphate flavone & & \\
\hline 81 & Hesperidin & \multirow{4}{*}{ FR of $\boldsymbol{H} . \boldsymbol{i}$. } & \multirow{4}{*}{$36,55,57$} \\
\hline 82 & Viscumside A & & \\
\hline 83 & 3',5,7,8-tetrahydroxy-4'-methoxyflavone 8- $O$ - $\beta$-d-glucopyranosiduronic acid methyl ester & & \\
\hline 84 & 4',5,7,8-tetrahydroxyflavone 8 - $O$ - $\beta$-D-glucopyranosiduronic acid methyl ester & & \\
\hline
\end{tabular}

Compounds Phenolics

\begin{tabular}{|c|c|c|c|}
\hline 85 & Rosmarinic acid & $\begin{array}{c}\text { RT of H.a., H.veg. } \\
\text { AP of H.e. }\end{array}$ & 36,55 \\
\hline$\overline{86}$ & 4'-O- $\beta$-D-glucopyranosyl rosmarinic acid & \multirow{3}{*}{ FR of $\boldsymbol{H} . \boldsymbol{i}$. } & \multirow{3}{*}{38} \\
\hline 87 & 4,4'-O-di- $\beta$-D-glucopyranosyl rosmarinic acid & & \\
\hline 88 & 4'-O- $\beta$-D-glucopyranosyl isorinic acid & & \\
\hline 89 & 3'-O-(8"'-Z-caffeoyl) rosmarinic acid & LV of H.veg. & 47 \\
\hline 90 & 3-(3,4-dimethoxyphenyl)-2-propenal & RT of $\boldsymbol{H} . \boldsymbol{a}$. & 51,52 \\
\hline 91 & Catechol & RT of $\boldsymbol{H} . \boldsymbol{i}$. & 24 \\
\hline 92 & 4-hydroxybenzoic acid & RT of $\boldsymbol{H} . \boldsymbol{h}$. & 54 \\
\hline
\end{tabular}


Table 2. Isolated compounds from Helicteres genus (cont.)

\begin{tabular}{|c|c|c|c|}
\hline $\mathrm{N}^{\circ}$ & Name & Source & Literature \\
\hline \multicolumn{4}{|c|}{ Compounds Phenolics } \\
\hline 93 & 3,4-dihydroxybenzoic acid methyl ester & \multirow{2}{*}{ RT of $\boldsymbol{H} . \boldsymbol{h}$. } & \multirow{2}{*}{54} \\
\hline 94 & 4-hydroxy-3,5-dimethoxybenzoic acid & & \\
\hline 95 & Syringic acid-4-O- $\alpha$-L-rhamnopyanoside & \multirow{2}{*}{ RT of $\boldsymbol{H} . \boldsymbol{a}$. } & \multirow{2}{*}{52} \\
\hline 96 & Protocatechuic aldehyde & & \\
\hline 97 & Gallic acid & RT of $\boldsymbol{H} . \boldsymbol{i}$. & 24 \\
\hline 98 & Vanillin & LV of $\boldsymbol{H} . \boldsymbol{i}$. & 24 \\
\hline 99 & Coniferyl alcohol & RT of $\boldsymbol{H} . \boldsymbol{a}$. & 52 \\
\hline 100 & Caffeic acid & RT of $\boldsymbol{H} . \boldsymbol{i}$. & 24 \\
\hline 101 & $p$-Coumaric acid & LV of $\boldsymbol{H} . \boldsymbol{i}$. & 24 \\
\hline 102 & Methyl caffeate & AP of $\boldsymbol{H} . \boldsymbol{h}$. & 54 \\
\hline \multicolumn{4}{|c|}{ Lignoids } \\
\hline 103 & Lariciresinol & \multirow{3}{*}{ RT of $\boldsymbol{H} . \boldsymbol{a}$. } & \multirow{3}{*}{51,57} \\
\hline 104 & Hedyotol C 7"-O- $\beta$-D-glucopyranoside & & \\
\hline 105 & Hedyotol D 7"- $O-\beta$-D-glucopyranoside & & \\
\hline 106 & (+)-pinoresinol & $\begin{array}{c}\text { RT of } \boldsymbol{H} . \boldsymbol{a} ., \boldsymbol{H} . \boldsymbol{h} . \\
\text { AP of } \boldsymbol{H} . \boldsymbol{v e l} .\end{array}$ & 12,46 \\
\hline$\overline{107}$ & (+/-)-medioresinol & \multirow{4}{*}{ RT of $\boldsymbol{H} . \boldsymbol{a}$. and $\boldsymbol{H} . \boldsymbol{h}$. } & \multirow{4}{*}{46,51} \\
\hline 108 & $(+/-)$-syringaresinol & & \\
\hline 109 & (-)-boehmenan & & \\
\hline 110 & (-)-boehmenan $\mathrm{H}$ & & \\
\hline 111 & (+/-)-trans-dihydrodiconiferyl alcohol & $\begin{array}{l}\text { RT of } \boldsymbol{H} . \boldsymbol{a} . \\
\text { FR of } \boldsymbol{H} . \boldsymbol{i} .\end{array}$ & $46,51,73$ \\
\hline 112 & $(7 S, 8 R)$-Urolignoside & RT of $\boldsymbol{H} . \boldsymbol{a}$. & 52 \\
\hline 113 & $(7 S, 8 R)$-Dihydrodehydrodiconiferyl alcohol & RT of $\boldsymbol{H} . \boldsymbol{a}$. and $\boldsymbol{H} . \boldsymbol{h}$. & 12,57 \\
\hline 114 & Helisorin & \multirow{9}{*}{ FR of $\boldsymbol{H} . \boldsymbol{i}$. } & \multirow{9}{*}{73,74} \\
\hline 115 & Helicterins C & & \\
\hline 116 & Helicterins E & & \\
\hline 117 & Helicterins D & & \\
\hline 118 & Helicterins F & & \\
\hline 119 & Helicterins A & & \\
\hline 120 & Helicterins B & & \\
\hline 121 & Helisterculins A & & \\
\hline 122 & Helisterculins B & & \\
\hline
\end{tabular}

\section{$123 \quad$ Perezone}

124 2,6-Dimethoxy-p-benzoquinone

125 8-acetyl-9-hydroxy-3-methoxy-7-methyl-1-phenalenon

126 Heliquinone

127 Heliquinone methyl ether

128 Mansonone F

RT of $\boldsymbol{H . a}$.

129 Mansonone E

130 Mansonone $\mathrm{H}$

131 Mansonone $\mathrm{M}$

132 6-[2-(5-acetyl-2,7-dimethyl-8-oxo-bicyclo[4.2.0]octa-1,3,5-trien-7-yl)-2-oxo-ethyl]-3,9-

132 dimethylnaphtho[1,8-bc]pyran-7,8-dione

\begin{tabular}{|c|c|c|c|}
\hline & Other compound & & \\
\hline 133 & Malatyamine ethyl ester (Amine) & \multirow{2}{*}{ RT of $\boldsymbol{H} . \boldsymbol{i}$. } & \multirow{2}{*}{$53,76-79$} \\
\hline 134 & Diosgenin (Saponin) & & \\
\hline 135 & 6,7-dihydroxy-3,8,11-trimethylcyclohexo-[d,e]-coumarin & \multirow{2}{*}{ RT of $\boldsymbol{H} . \boldsymbol{a}$. } & \multirow{2}{*}{51,64} \\
\hline 136 & 6,7,9 $\alpha$-trihydroxy-3,8,11 $\alpha$-trimethylcyclohexo-[d,e]-coumarin (Coumarin) & & \\
\hline 137 & Tetratriacontanol (Alcohol) & \multirow{2}{*}{ LV of $\boldsymbol{H} . \boldsymbol{i}$. } & \multirow{2}{*}{67} \\
\hline 138 & Tetratriacontanoic acid (Fatty acid) & & \\
\hline 139 & Palmitic acid (Fatty acid) & $\begin{array}{l}\text { LV of } \boldsymbol{H} . \boldsymbol{i} . \\
\text { AP of } \boldsymbol{H} \cdot \boldsymbol{v e l}\end{array}$ & 12,24 \\
\hline$\underline{140}$ & Aliphatic alcohol decanol (Alcohol) & AP of H.vel. and H.e. & 12,48 \\
\hline 141 & Helicterone A (Alkaloid) & RT of $\boldsymbol{H} . \boldsymbol{a}$. & 52 \\
\hline 142 & Yohimbine (Alkaloid) & FR of $\boldsymbol{H} . \boldsymbol{i}$. & 80 \\
\hline 143 & Pheophytin A & RT of $\boldsymbol{H} . \boldsymbol{i}$. & \multirow{2}{*}{12,81} \\
\hline 144 & Pheophytin B & AP of $\boldsymbol{H} \cdot \boldsymbol{v}$ el. & \\
\hline
\end{tabular}


Table 2. Isolated compounds from Helicteres genus (cont.)

\begin{tabular}{|c|c|c|c|}
\hline $\mathrm{N}^{\circ}$ & Name & Source & Literature \\
\hline 145 & $13^{2}$-hydroxy-(132-R)-pheophytin a & \multirow{2}{*}{ AP of $\boldsymbol{H} . v e l$. } & \multirow{2}{*}{12} \\
\hline 146 & $13^{2}$-hydroxy- $\left(13^{2}-S\right)$-pheophytin a & & \\
\hline 147 & Ellagic acid (Tannin) & RT of $\boldsymbol{H} . \boldsymbol{i}$. & 82,83 \\
\hline 148 & 3,6,9-trimethyl-pyrano[2,3,4-de]chromen-2-one (Lactone) & RT of $\boldsymbol{H} . \boldsymbol{a}$. & 75 \\
\hline 149 & 4-4'-sulfinylbis(2-(tert-butyl)-5-methylphenol & AP of $\boldsymbol{H} . \boldsymbol{h}$. & 56,70 \\
\hline
\end{tabular}

H.i.: H. isora; H.a.: H. angustifolia; H.vel.: H. velutina; H.h.: H. hirsuta; H.veg.: H. vegae; H.e.: H. eichleri. RT: Roots; AP: Aerial Parts; FR: Fruits; LV: Leaves.

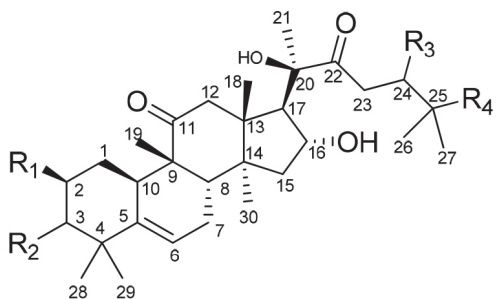

1. $\mathrm{R}_{1}: H ; \mathrm{R}_{2}:=\mathrm{O} ; \mathrm{R}_{3}: \mathrm{H} ; \mathrm{R}_{4}: \mathrm{OCOCH}_{3} ; \Delta^{23,24}$

2. $\mathrm{R}_{1}: \mathrm{HSO}_{3} ; \mathrm{R}_{2}: \mathrm{H} ; \mathrm{R}_{3}: \mathrm{H} ; \mathrm{R}_{4}: \mathrm{OCOCH}_{3} ; \Delta^{23,24}$

3. $\mathrm{R}_{1}:=\mathrm{O} ; \mathrm{R}_{2}: \mathrm{OH} ; \mathrm{R}_{3}: \mathrm{H} ; \mathrm{R}_{4}: \mathrm{OCOCH}_{3} ; \Delta^{23,24}$

4. $\mathrm{R}_{1}: \mathrm{OH} ; \mathrm{R}_{2}:=\mathrm{O} ; \mathrm{R}_{3}: \mathrm{H} ; \mathrm{R}_{4}: \mathrm{OH} ; \Delta^{23,24}$

5. $\mathrm{R}_{1}: \mathrm{OH} ; \mathrm{R}_{2}:=\mathrm{O} ; \mathrm{R}_{3}: \mathrm{H} ; \mathrm{R}_{4}: \mathrm{OCOCH}_{3} ; \Delta^{23,24} ; \Delta^{1,2}$

6. $\mathrm{R}_{1}$ : OGIc; $\mathrm{R}_{2}:=\mathrm{O} ; \mathrm{R}_{3}: \mathrm{OH} ; \mathrm{R}_{4}: \mathrm{OH}$

7. $\mathrm{R}_{1}: \mathrm{OH} ; \mathrm{R}_{2}:=\mathrm{O} ; \mathrm{R}_{3}: \mathrm{H} ; \mathrm{R}_{4}: \mathrm{OH} ; \Delta^{23,24} ; \Delta^{1,2}$

8. $\mathrm{R}_{1}: \mathrm{OH} ; \mathrm{R}_{2}:=\mathrm{O} ; \mathrm{R}_{3}: \mathrm{OH} ; \mathrm{R}_{4}: \mathrm{OH} ; \Delta^{1,2}$

9. $\mathrm{R}_{1}:=\mathrm{O} ; \mathrm{R}_{2}: \mathrm{OH} ; \mathrm{R}_{3}: \mathrm{H} ; \mathrm{R}_{4}: \mathrm{OH} ; \Delta^{23,24}$

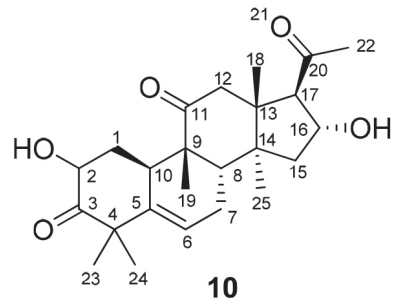<smiles>CC(C)C1CCC2[C@H]3CC[C@]4(C)C5CCC(O)C(C)(C)C5=CC[C@H]4[C@]3(C)CC[C@]12C</smiles>

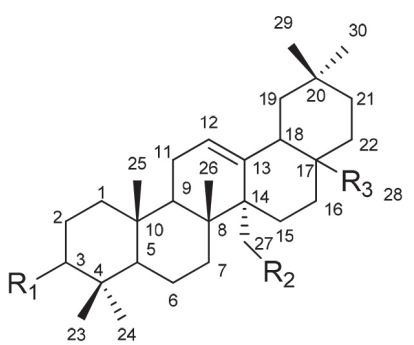

35. $\mathrm{R}_{1}: \mathrm{OH} ; \mathrm{R}_{2}: \mathrm{OAc} ; \mathrm{R}_{3}: \mathrm{H}$

36. $\mathrm{R}_{1}: \mathrm{OH} ; \mathrm{R}_{2}$ : benzoyl; $\mathrm{R}_{3}: \mathrm{COOCH}_{3}$

37. $\mathrm{R}_{1}$ : O-cinnamoyl; $\mathrm{R}_{2}: \mathrm{H} ; \mathrm{R}_{3}: \mathrm{COOH}$

38. $\mathrm{R}_{1}$ : OAc; $\mathrm{R}_{2}$ : O-4-hydroxybenzoyl; $\mathrm{R}_{3}$ : OAc

39. $\mathrm{R}_{1}$ : OAc; $\mathrm{R}_{2}$ : O-4-hydroxybenzoyl; $\mathrm{R}_{3}: \mathrm{COOCH}_{3}$

40. $\mathrm{R}_{1}$ : OAc; $\mathrm{R}_{2}$ : O-PhCO; $\mathrm{R}_{3}: \mathrm{COOCH}_{3}$

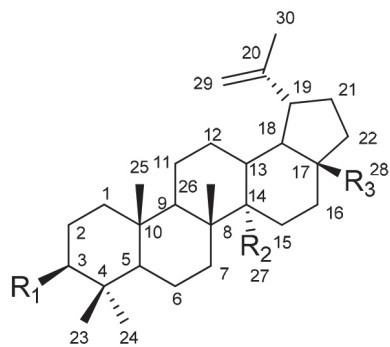

11. $\mathrm{R}_{1}: \mathrm{OH} ; \mathrm{R}_{2}: \mathrm{CH}_{3} ; \mathrm{R}_{3}: \mathrm{H}$

12. $\mathrm{R}_{1}: \mathrm{OH} ; \mathrm{R}_{2}: \mathrm{CH}_{3} ; \mathrm{R}_{3}: \mathrm{COOH}$

,2 13. $\mathrm{R}_{1}$ :O-benzoyl; $\mathrm{R}_{2}: \mathrm{CH}_{3} ; \mathrm{R}_{3}: \mathrm{COOH}$

14. $\mathrm{R}_{1}: \mathrm{OH} ; \mathrm{R}_{2}: \mathrm{CH}_{3} ; \mathrm{R}_{3}: \mathrm{COOCH}_{3}$

15. $\mathrm{R}_{1}$ : O-trans-coumaroyl; $\mathrm{R}_{2}: \mathrm{CH}_{3} ; \mathrm{R}_{3}: \mathrm{COOH}$

16. $\mathrm{R}_{1}$ : O-trans-caffeoyl; $\mathrm{R}_{2}: \mathrm{CH}_{3} ; \mathrm{R}_{3}: \mathrm{COOH}$

17. $\mathrm{R}_{1}$ : O-trans-feruloyl; $\mathrm{R}_{2}: \mathrm{CH}_{3} ; \mathrm{R}_{3}: \mathrm{COOH}$

18. $\mathrm{R}_{1}$ : O-trans-coumaroyl; $\mathrm{R}_{2}: \mathrm{CH}_{3} ; \mathrm{R}_{3}: \mathrm{CH}_{2} \mathrm{OH}$

19. $\mathrm{R}_{1}$ : O-cis-coumaroyl; $\mathrm{R}_{2}: \mathrm{CH}_{3} ; \mathrm{R}_{3}: \mathrm{CH}_{2} \mathrm{OH}$

20. $\mathrm{R}_{1}$ : O-trans-caffeoyl; $\mathrm{R}_{2}: \mathrm{CH}_{3} ; \mathrm{R}_{3}: \mathrm{CH}_{2} \mathrm{OH}$

21. $R_{1}$ : O-trans-feruloyl; $\mathrm{R}_{2}: \mathrm{CH}_{3} ; \mathrm{R}_{3}: \mathrm{CH}_{2} \mathrm{OH}$

22. $\mathrm{R}_{1}: \mathrm{OCOCH}_{3} ; \mathrm{R}_{2}: \mathrm{CH}_{3} ; \mathrm{R}_{3}: \mathrm{COOH}$

23. $\mathrm{R}_{1}: \mathrm{OCOCH}_{3} ; \mathrm{R}_{2}: \mathrm{CH}_{3} ; \mathrm{R}_{3}: \mathrm{CH}_{2} \mathrm{OH}$

24. $R_{1}=R_{2}: O A C ; R_{3}: C O O H$

25. $R_{1}$ : OAc; $R_{2}$ : benzoyloxy; $R_{3}: C O O H$

26. $\mathrm{R}_{1}$ : $\mathrm{OAc} ; \mathrm{R}_{2}=\mathrm{H} ; \mathrm{R}_{3}=\mathrm{OH}$

27. $R_{1}$ : caffeate; $R_{2}=H ; R_{3}=\mathrm{COOH}$

28. $\mathrm{R}_{1}$ : OH; $\mathrm{R}_{2}$ :benzoyloxy; $\mathrm{R}_{3}=\mathrm{COOH}$

29. $\mathrm{R}_{1}: \mathrm{OH} ; \mathrm{R}_{2}$ : benzoyloxy; $\mathrm{R}_{3}=\mathrm{COOCH}_{3}$

30. $\mathrm{R}_{1}$ : OCOOH; $\mathrm{R}_{2}$ : $\mathrm{CH}_{2}$-benzoyloxy; $\mathrm{R}_{3}=\mathrm{COOCH}_{3}$

31. $\mathrm{R}_{1}$ : OAc; $\mathrm{R}_{2}=\mathrm{CH}_{2}$-O-trans-cinnamoyl; $\mathrm{R}_{3}=\mathrm{COOCH}_{3}$

32: $\mathrm{R}_{1}$ : OAc; $\mathrm{R}_{2}=\mathrm{CH}_{2}-\mathrm{O}$-4-hydroxybenzoyl; $\mathrm{R}_{3}=\mathrm{COOH}$

33: $\mathrm{R}_{1}: \mathrm{OH} ; \mathrm{R}_{2}=\mathrm{CH}_{2} \mathrm{OH} ; \mathrm{R}_{3}=\mathrm{COOH}$
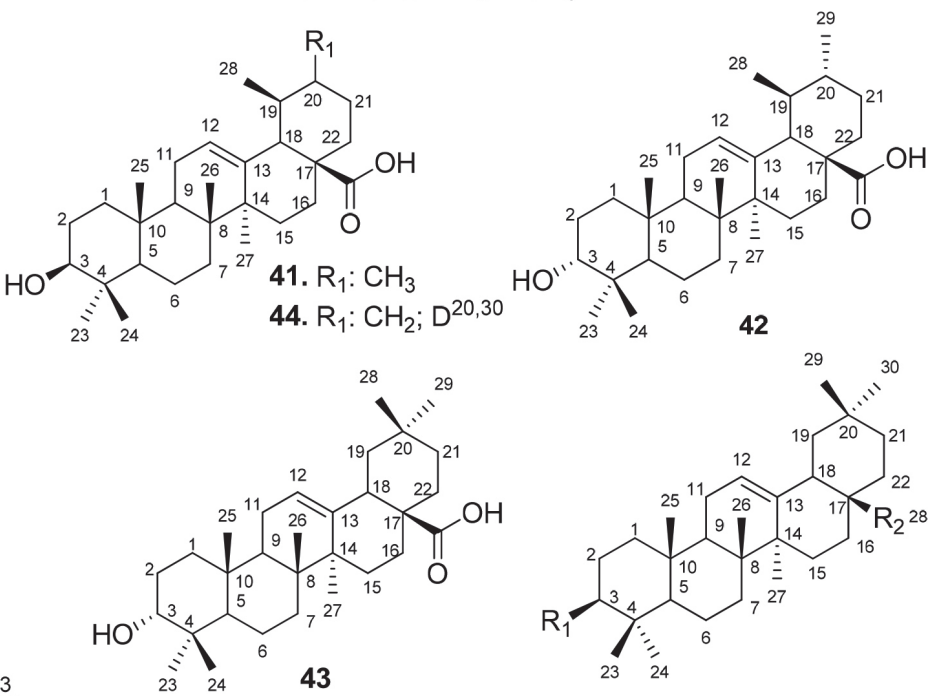

45. $\mathrm{R}_{1}: \mathrm{OH} ; \mathrm{R}_{2}: \mathrm{COOH}$

46. $\mathrm{R}_{1}: \mathrm{OCOCH}_{3} ; \mathrm{R}_{2}: \mathrm{COOH}$

47. $R_{1}$ : Phytil; $R_{2}: H$

Figure 2. Compounds isolated from Helicteres species 


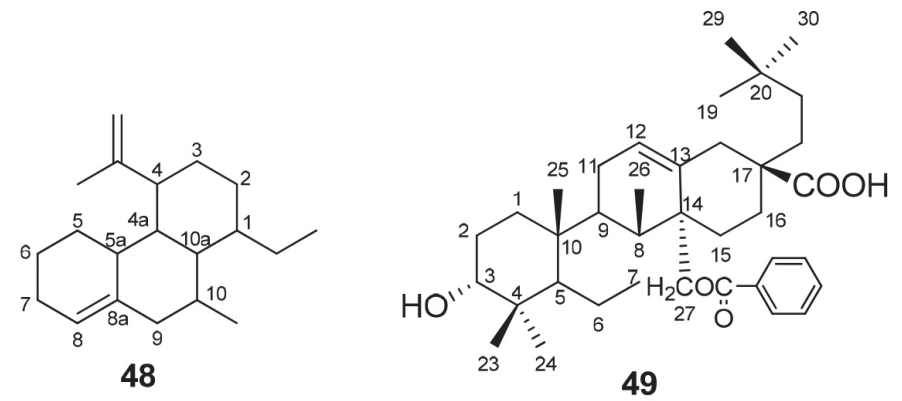

49

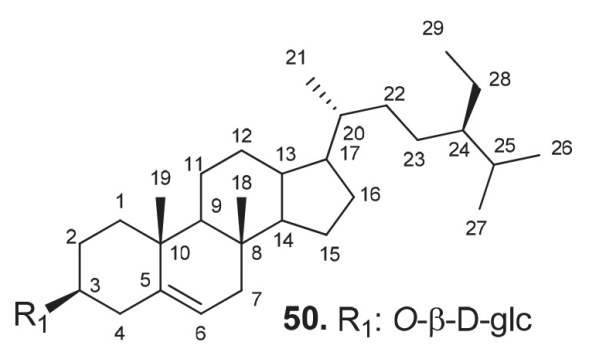

51. $\mathrm{R}_{1}: \mathrm{OH}$

52. $\mathrm{R}_{1}: \mathrm{OH} ; \Delta^{22,23}$

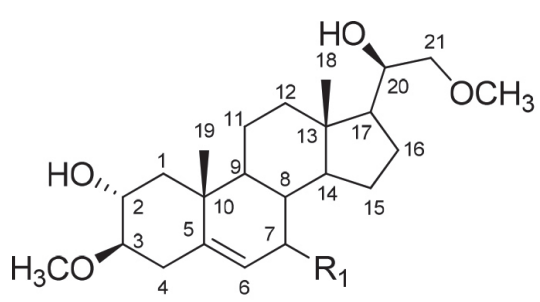

53. $\mathrm{R}_{1}:=\mathrm{O}$

54. $\mathrm{R}_{1}: \mathrm{OH}$

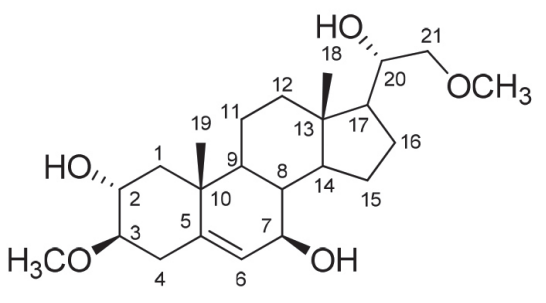

55<smiles>[R]c1cc(C2Oc3c([R])c([R])c([R])c(O)c3C(=O)C2[R4])cc([R])c1[R]</smiles>

57. $\mathrm{R}_{1}=\mathrm{R}_{3}=\mathrm{R}_{5}=\mathrm{R}_{7}: \mathrm{H} ; \mathrm{R}_{2}=\mathrm{R}_{6}: O H ; \mathrm{R}_{4}: O-\beta-\mathrm{D}$-galactoside; $\Delta^{2,3}$

58. $R_{1}: O-\beta$-D-galactoside; $R_{3}=R_{5}=R_{7}: H ; R_{2}=R_{6}=R_{4}: O H ; \Delta^{2,3}$

59. $R_{1}=R_{6}: O H ; R_{2}: O_{3} ; R_{3}=R_{4}=R_{5}=R_{7}: H ; \Delta^{2,3}$

60. $\mathrm{R}_{1}: \mathrm{OH} ; \mathrm{R}_{2}: \mathrm{OCH}_{3} ; \mathrm{R}_{3}=\mathrm{R}_{4}=\mathrm{R}_{5}=\mathrm{R}_{7}: \mathrm{H} ; \mathrm{R}_{6}: \mathrm{OCH}_{3} ; \Delta^{2,3}$

61. $\mathrm{R}_{1}$ : O- $\beta$-D-glucupyranoside; $\mathrm{R}_{2}: \mathrm{OH} ; \mathrm{R}_{3}=\mathrm{R}_{4}=\mathrm{R}_{7}: \mathrm{H} ; \mathrm{R}_{6}: \mathrm{OCH}_{3} ; \Delta^{2,3}$

62. $\mathrm{R}_{1}$ : O- $\beta$-D-glcuronide, 6" $n$-buthyl ester; $\mathrm{R}_{2}: \mathrm{OH} ; \mathrm{R}_{3}=\mathrm{R}_{4}=\mathrm{R}_{5}=\mathrm{R}_{7}: \mathrm{H} ; \mathrm{R}_{6}: \mathrm{OCH}_{3} ; \Delta^{2,3}$

63. $R_{1}$ : O- $\beta$-D-glcuronide; $\mathrm{R}_{2}: \mathrm{OH} ; \mathrm{R}_{3}=\mathrm{R}_{4}=\mathrm{R}_{5}=\mathrm{R}_{7}: \mathrm{H} ; \mathrm{R}_{6}: \mathrm{OCH}_{3} ; \Delta^{2,3}$

64. $R_{1}$ : O- $\beta$-D-glcuronide, 2 " sulphate; $R_{2}: O H ; R_{3}=R_{4}=R_{5}=R_{7}: H ; R_{6}: O C H_{3} ; \Delta^{2,3}$

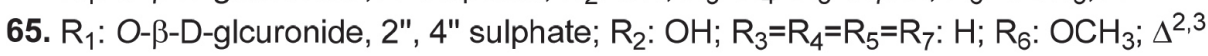

66. $R_{1}$ : O- $\beta$-D-glcuronide, 2", 4" sulphate; $R_{2}=R_{6}: O H ; R_{3}=R_{4}=R_{5}=R_{7}: H ; \Delta^{2,3}$

67. $R_{1}=R_{3}=R_{5}=R_{7}: H ; R_{2}=R_{6}: O H ; R_{4}: O-\beta-D-g l c ; \Delta^{2,3}$

68. $R_{1}=R_{3}=R_{5}=R_{7}: H ; R_{2}=R_{4}=R_{6}: O H ; \Delta^{2,3}$

69. $R_{1}: H ; R_{2}=R_{6}: O H ; R_{3}: H ; R_{4}:$ O-glc-6"-p-coumaroyl; $R_{5}=R_{7}: \Delta^{2,3}$

70. $R_{1}=R_{2}: O H ; R_{3}=R_{4}=R_{5}=R_{7}: H ; R_{6}: O C H_{3} ; \Delta^{2,3}$

71. $\mathrm{R}_{1}=\mathrm{R}_{2}=\mathrm{R}_{7}: \mathrm{OH} ; \mathrm{R}_{3}=\mathrm{R}_{4}=\mathrm{R}_{5}: \mathrm{H} ; \mathrm{R}_{6}: \mathrm{OCH}_{3} ; \Delta^{2,3}$

72. $R_{1}$ : O- $\beta$-D-glucuronide, 6" methyl ester; $\mathrm{R}_{2}: \mathrm{OH} ; \mathrm{R}_{3}=\mathrm{R}_{4}=\mathrm{R}_{7}: \mathrm{H} ; \mathrm{R}_{6}: \mathrm{OCH}_{3} ; \Delta^{2,3}$

73. $\mathrm{R}_{1}$ : O- $\beta$-D-glucuronide, 2" sodium sulphate; $\mathrm{R}_{2}: \mathrm{OH} ; \mathrm{R}_{3}=\mathrm{R}_{4}=\mathrm{R}_{7}: \mathrm{H} ; \mathrm{R}_{6}: \mathrm{OCH}_{3} ; \Delta^{2,3}$

74. $R_{1}: O-\beta-D$-glucuronide; $R_{2}: O H ; R_{3}=R_{4}=R_{7}: H ; R_{6}: O_{3} ; \Delta_{3} 2,3$

75. $R_{1}$ : O- $\beta$-D-glucuronide; $R_{3}=R_{4}=R_{5}=R_{7}: H ; R_{2}=R_{7}: O H ; R_{6}: O C H_{3} ; \Delta^{2,3}$

76. $R_{1}: O H ; R_{2}=R_{6}: O C H_{3} ; R_{3}=R_{4}=R_{5}=R_{7}: H ; \Delta^{2,3}$

77. $R_{1}=R_{3}=R_{4}: H ; R_{2}=R 6: O H ; R_{5}=R_{7}: O_{3} ; \Delta^{2,3}$

78. $\mathrm{R}_{1}: \mathrm{OSO}_{3} \mathrm{H} ; \mathrm{R}_{2}=\mathrm{R}_{6}: O \mathrm{OCH}_{3} ; \mathrm{R}_{3}=\mathrm{R}_{4}=\mathrm{R}_{5}=\mathrm{R}_{7}: \mathrm{H} ; \Delta^{2,3}$

79. $\mathrm{R}_{1}: \mathrm{OSO}_{3} \mathrm{H} ; \mathrm{R}_{2}: \mathrm{OCH}_{3} ; \mathrm{R}_{3}=\mathrm{R}_{4}=\mathrm{R}_{5}=\mathrm{R}_{7}: \mathrm{H} ; \mathrm{R}_{6}: O H ; \Delta^{2,3}$

80. $\mathrm{R}_{1}: \mathrm{OSO}_{3} \mathrm{H} ; \mathrm{R}_{2}=\mathrm{R}_{6}: \mathrm{OCH}_{3} ; \mathrm{R}_{3}: \mathrm{OH} ; \mathrm{R}_{4}=\mathrm{R}_{5}=\mathrm{R}_{7}: \mathrm{H} ; \Delta^{2,3}$

81. $R_{1}=R_{3}=R_{4}=R_{5}: H ; R_{2}:$ O-rhamnosyl, $6^{\prime \prime}$ glc; $R_{6}: O H ; R_{7}: O_{7}$

82. $R_{1}=R_{3}=R_{4}=R_{5}: H ; R_{2}: O-\beta$-D-glc; $R_{6}: O H ; R_{7}: O_{3}$

83. $R_{1}$ : O- $\beta$-D-glucopyranosiduronic acid methyl ester; $R_{3}=R_{4}=R_{5}: H ; R_{6}: O C H 3 ; R_{7}: O H$

84. $R_{1}$ : O- $\beta$-D-glucopyranosiduronic acid methyl ester; $R_{3}=R_{4}=R_{5}=R_{7}: H ; R_{6}: O H$; 
<smiles>[R6]Oc1cc(C[C@@H](OC(=O)/C=C/c2ccc(O)c(O[R6])c2)C(=O)O)ccc1[R2]</smiles>

85. $\mathrm{R}_{1}=\mathrm{R}_{3}: \mathrm{H} ; \mathrm{R}_{2}=\mathrm{OH}$;

86. $\mathrm{R}_{1}: H ; \mathrm{R}_{2}: \mathrm{OH} ; \mathrm{R}_{3}: \beta-D-g l u c$

87. $\mathrm{R}_{1}: \beta$-D-glc; $\mathrm{R}_{2}: \mathrm{OH} ; \mathrm{R}_{3}: \beta$-D-glc

88. $R_{1}=R_{2}: H ; R_{3}: \beta$-D-gluc

89. $\mathrm{R}_{1}: \mathrm{H} ; \mathrm{R}_{2}: \mathrm{OH} ; \mathrm{R}_{3}$ : caffeoyl

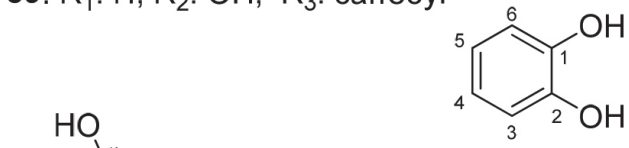<smiles></smiles>
91

103<smiles>COc1cc(C(=O)[C@@H](CO)Oc2c(OC)cc([C@@H]3OCC4CCCCC43)cc2OC)ccc1O</smiles>

113. $R_{1}$ : Glc

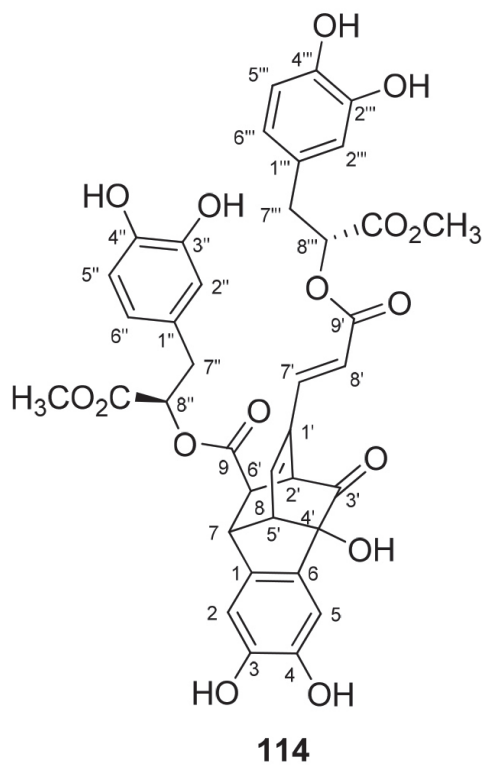

112. $\mathrm{R}_{1}: \mathrm{OH}$<smiles>[R12]OCc1cc(OC)c2c(c1)[C@H](CO)[C@H]([Z6]1ccc([R])c(OC)c1)O2</smiles><smiles>[R]C(=O)C=Cc1ccc([R3])c([R])c1</smiles>

90. $\mathrm{R}_{1}: \mathrm{H} ; \mathrm{R}_{2}=\mathrm{R} 3: \mathrm{OCH}_{3}$ 100. $\mathrm{R}_{1}=\mathrm{R}_{3}: \mathrm{OH} ; \mathrm{R}_{2}=\mathrm{H}$

101. $R_{1}=R_{2}=R_{3}: O H$

102. $\mathrm{R}_{1}: \mathrm{OCH}_{3} ; \mathrm{R}_{2}=\mathrm{R}_{3}: \mathrm{OH}$<smiles>[R]C(=O)c1cc([R])c([R4])c([R])c1</smiles>

92. $\mathrm{R}_{1}=\mathrm{R}_{4}: \mathrm{OH} ; \mathrm{R}_{2}=\mathrm{R}_{3}: \mathrm{H}$

93. $\mathrm{R}_{1}: \mathrm{OCH}_{3} ; \mathrm{R}_{2}: \mathrm{HR}_{3}=\mathrm{R}_{4}: \mathrm{OH}$

94. $\mathrm{R}_{1}=\mathrm{R}_{4}: \mathrm{OH} ; \mathrm{R}_{2}=\mathrm{R}_{3}: \mathrm{OCH}_{3}$

95. $\mathrm{R}_{1}: \mathrm{OH} ; \mathrm{R}_{2}=\mathrm{R}_{3}: \mathrm{OCH}_{3} ; \mathrm{R}_{4}: \alpha-L-O-R h a m n o s y l$

96. $\mathrm{R}_{1}=\mathrm{R}_{2}: \mathrm{H} ; \mathrm{R}_{3}=\mathrm{R}_{4}: \mathrm{OH}$

97. $\mathrm{R}_{1}: \mathrm{H} ; \mathrm{R}_{2}=\mathrm{R}_{3}=\mathrm{R}_{4}: \mathrm{OH}$

98. $\mathrm{R}_{1}=\mathrm{R}_{2}: \mathrm{H} ; \mathrm{R}_{3}: \mathrm{OCH}_{3} ; \mathrm{R}_{4}: \mathrm{OH}$<smiles>COc1cc(CCCO)ccc1O</smiles><smiles>[R2]c1cc([C]2OC[C@H]3[C@@H]2CO[C@H]3c2cc([R2])c([R4])c(OC)c2)cc(OC)c1[R]</smiles>

106. $\mathrm{R}_{1}=\mathrm{R}_{4}: \mathrm{OH} ; \mathrm{R}_{2}=\mathrm{R}_{3}: \mathrm{OH}$

107. $\mathrm{R}_{1}=\mathrm{R}_{4}: \mathrm{OH} ; \mathrm{R}_{2}: \mathrm{OCH}_{3} ; \mathrm{R}_{3}: \mathrm{H}$ 108. $R_{1}=R_{4}: O H ; R_{2}=R_{3}: O C H_{3}$

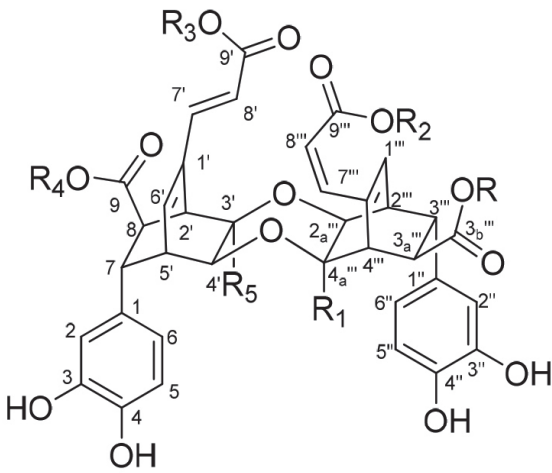

115. $\mathrm{R}_{1}=\mathrm{R}_{5}: \mathrm{OCH}_{3} ; \mathrm{R}: \mathrm{CH}_{3} ; ; \mathrm{R}_{2}=\mathrm{R}_{3}=\mathrm{R}_{4}: X$ 116. $\mathrm{R}_{1}: \mathrm{OCH}_{3} ; \mathrm{R}: \mathrm{CH}_{3} ; \mathrm{R}_{2}=\mathrm{R}_{3}=\mathrm{R}_{4}: \mathrm{X} ; \mathrm{R}_{5}: \mathrm{OH}$

117. $R_{1}=R_{5}: O C H_{3} ; R_{2}: C H_{3} ; ; R=R_{3}=R_{4}: X$

118. $\mathrm{R}_{1}: \mathrm{OH} ; \mathrm{R}_{2}: \mathrm{CH}_{3} ; \mathrm{R}=\mathrm{R}_{3}=\mathrm{R}_{4}: \mathrm{X} ; \mathrm{R}_{5}: \mathrm{OCH}_{3}$

119. $R_{1}=R_{5}: O C H_{3} ; R=R_{2}=R_{3}=R_{4}: X$

120. $R_{1}=O H ; R=R_{2}=R_{3}=R_{4}: X ; R_{5}: O C H_{3}$

$\mathrm{X}$.

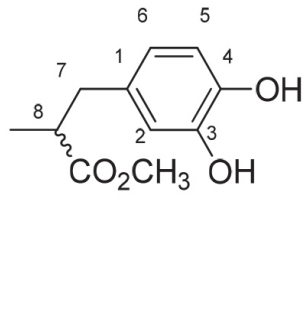

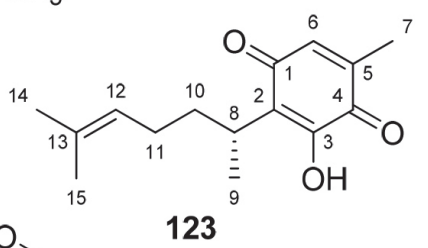

123

Figure 2. Compounds isolated from Helicteres species (cont.) 
<smiles>COC1=CC(=O)c2c(O)c(C(C)=O)c(C)c3cccc1c23</smiles>

125<smiles>[R1]c1cc(C)c(C)c2c1C(=O)C(OCC(C)C)=C(C)C2=O</smiles>

126. $\mathrm{R}_{1}: \mathrm{OH}$ 127. $\mathrm{R}_{1}: \mathrm{OCH}_{3}$<smiles>[R]c1cc(C)c2c3c1[C@H](C)COC3=C(C)C(=O)C2=O</smiles>

128. $R_{1}: H ; \Delta^{2,3}$

129. $\mathrm{R}_{1}: \mathrm{H}$

130. $\mathrm{R}_{1}: \mathrm{OH}$

131. $\mathrm{R}_{1}: \mathrm{OCH}_{3}$<smiles></smiles><smiles>[R]CCCCCCCCCC[R]</smiles>

137. $\mathrm{R}_{1}: \mathrm{OH} ; \mathrm{R}_{2}:\left(\mathrm{CH}_{2}\right)_{24} \mathrm{CH}_{3}$

138. $\mathrm{R}_{1}: \mathrm{COOH} ; \mathrm{R}_{2}:\left(\mathrm{CH}_{2}\right)_{23} \mathrm{CH}_{3}$

139. $\mathrm{R}_{1}: \mathrm{COOH} ; \mathrm{R}_{2}:\left(\mathrm{CH}_{2}\right)_{5} \mathrm{CH}_{3}$

140. $\mathrm{R}_{1}: \mathrm{OH} ; \mathrm{R}_{2}: \mathrm{CH}_{3}$<smiles></smiles>

141<smiles>[Y10]C1[C@@H]2CC[C@H](O)[C@@H](C(=O)OC)[C@H]2C[C@H]2c3[nH]c4ccccc4c3CCN12</smiles>

147<smiles>CC1=COc2c(C)c(=O)oc3c(C)ccc1c23</smiles>

148<smiles>Cc1cc(O)c(C(C)(C)C)cc1S(=O)c1cc(C(C)(C)C)c(O)cc1C</smiles>

149

Figure 2. Compounds isolated from Helicteres species (cont.)

\section{Anti-inflammatory and analgesic activity}

Natural products are widely used in folk medicine to treat inflammatory conditions, including fever, pain, migraine and arthritis, being targets for the development of new anti-inflammatory drugs. ${ }^{172}$ Studies with plant extracts have shown promissory activity. ${ }^{173}$

H. isora root extract showed antinociceptive activity in mice. ${ }^{101}$ $H$. angustifolia n-butanol fraction has anti-inflammatory and analgesic activity. ${ }^{104}$ Non-clinical mice studies have showed through photoacoustic spectroscopy that $H$. gardneriana extract induces a significant reduction in the inflamed area. ${ }^{106}$ Studies with extracts from the aerial parts of $H$. hirsute has been conducted in order to discover their mechanisms of action against inhibition of COX1 and COX2 in vitro ${ }^{70}$ (Table 3 ).
Antitumor and cytotoxic activity

Cancer is one of the leading causes of mortality in the world. About $60 \%$ of current anticancer drugs are from natural origin, with emphasis on plant species that are rich in anticancer agents and can be used as an alternative to chemotherapeutic drugs as they are less toxic. ${ }^{174}$ The effects of plant-derived natural products have been investigated to a large extent on cancer cell proliferation, survival, invasion and metastasis due to bioactivity and the diversity of their chemical constituents. ${ }^{60,175}$

Helicteres are used to decrease tumor progression by folk medicine. In order to evaluate this activity, extracts, fractions and isolated substances have been studied through the evaluation of cytotoxic activity mainly in liver, lung, colon and breast cancers (Table 3). 
Table 3. In vitro, in vivo, ex vivo and in silico biological studies reported from Helicteres genus

\begin{tabular}{|c|c|c|c|}
\hline Species & Material used & Experimental model & Literature \\
\hline \multicolumn{4}{|c|}{ Anti-inflammatory and Analgesic Activity } \\
\hline H.i. & Roots extracts & in vivo - antinociceptive & 101 \\
\hline H.a. & Ethyl acetate extract & in vivo - anti-inflammatory and antipyretic & 102,103 \\
\hline H.a. & $n$-butanolic fraction & in vivo - anti-inflammatory and analgesic & 104 \\
\hline H.h. & Aerial parts extract & in vitro - COX1/COX2 inhibition & 70 \\
\hline H.ga. & Aerial parts ethanolic extract & in vivo - acute inflammation & 105 \\
\hline H.ga. & Leaves ethanolic extract & ex vivo - topical use & 106 \\
\hline \multicolumn{4}{|c|}{ Antitumor and Cytotoxic Activity } \\
\hline H.i. & Fruits extract & in vitro - lung cancer & 107,108 \\
\hline H.i. & Curcubitacin B and Isocurcubitacin B & not reported & 109 \\
\hline H.i. & Stem hydroethanolic extract & in vivo - hepatocellular carcinoma & 110 \\
\hline H.a. & Roots aqueous extract & in vitro - fibroblasts and osteosarcoma & 111 \\
\hline H.a. & Triterpenes & in vitro - colorectal cancer & 60 \\
\hline H.a. & Roots aqueous extract & in vivo - human fibrosarcoma & 25 \\
\hline H.a. & Polysaccharides & in vivo - breast cancer & 112 \\
\hline H.a. & $\begin{array}{c}2,3,3 \beta-O-[(\mathrm{E}) \text {-coumaroyl] betulinic acid and pyracrenic } \\
\text { acid }\end{array}$ & in vitro - colorectal cancer & 44 \\
\hline H.a. & Roots ethanolic and aqueous extract & in vitro - cell lines of lung cancer, colon and hepatocellular carcinoma & 25,111 \\
\hline H.a. & Roots aqueous extract & $\begin{array}{l}\text { in vitro - osteosarcoma and in vivo - pulmonary metastasis and subcu- } \\
\text { taneous xenograft }\end{array}$ & 113 \\
\hline H.a. & Curcubitacin $\mathrm{B}$ and $\mathrm{J}$ & in vitro - hepatocellular carcinoma and malignant carcinoma & 51 \\
\hline H.h. & $(+/-)$-pinoresinol & in vitro - lung and breast cancer & 46 \\
\hline H.h. & Roots extract & in vitro - human $\mathrm{KB}$ cell lines & 114 \\
\hline H.veg. & Leaves extract & in vitro - Salmonella typhimurium & 47 \\
\hline H.s. & Leaves hydroethanolic extract & in vivo - ovarian cancer cell lineages & 8 \\
\hline \multicolumn{4}{|c|}{ Hepatoprotective Activity } \\
\hline H.i. & Bark aqueous extract & in vivo - changes in liver enzymes & 115 \\
\hline H.i. & Bark ethanolic extract & in vivo - changes in liver enzymes & $116-118$ \\
\hline H.a. & Aqueous extract & in vivo - inhibits hepatic fibrosis & 119 \\
\hline H.a. & Methyl helicterate & in vivo - inhibits hepatic fibrosis & 120,121 \\
\hline \multicolumn{4}{|c|}{ Antidiabetic and Hypolipidemic Activity } \\
\hline$\overline{H . i .}$ & Roots ethanolic extract & in vivo - sensitizing and hypolipidemic insulin & 122 \\
\hline H.i. & $n$-butanolic fraction & in vivo - maintain normal glycemic levels & 123 \\
\hline H.i. & Bark aqueous extract & in vivo - reduced blood glucose levels & 30 \\
\hline H.i. & Bark aqueous extract & in vivo - reduction of peroxidation products & 32 \\
\hline H.i. & Roots $n$-butanolic extract & in vivo - reduced glucose and total cholesterol & 124 \\
\hline H.i. & Bark aqueous extract & in vivo - reduction of cholesterol, free fatty acids and triglycerides & 31 \\
\hline H.i. & $n$-butanolic extract & in vivo - hypoglycemia & 125 \\
\hline H.i. & Fraction rich in saponin & in vivo - decreased serum levels of lipids and glucose & 126 \\
\hline H.i. & Stem extract & in vivo - glycogen and carbohydrate metabolism & 127 \\
\hline H.i. & Bark aqueous extract & in vivo - decreased glucose levels & 128 \\
\hline H.i. & Bark aqueous extract & in vivo - total blood glucose and lipids & 127 \\
\hline H.i. & Fruits aqueous extract & in vitro - glucose uptake & 129 \\
\hline H.i. & Fraction rich in saponin & in vitro - increases glycogen synthesis & 130 \\
\hline H.i. & Fruits ethanolic extract & in vivo - stabilizes lipids levels & 122 \\
\hline H.i. & Roots hydroethanolic extract and roots $n$-butanolic extract & in vivo - reduced glycemia, total cholesterol, triglycerides and urea & 34 \\
\hline H.i. & Fruits aqueous extract & in vivo - increases glucose uptake and transport & 131 \\
\hline H.i. & Stem extract & in vivo - normalizes glucose, urea and creatinine levels & 88,116 \\
\hline H.i. & Bark aqueous extract & in vivo - antiperoxidative activity & 88 \\
\hline H.i. & Isolated constituents & in silico - insulin receptors & 79 \\
\hline H.i. & Roots extract & in vivo - reduction of plasma glycoproteins & 132,133 \\
\hline H.i. & Roots extract & $\begin{array}{l}\text { in vivo and in vitro - inhibition of } \alpha \text {-amylase and reduction of gluta- } \\
\text { thione }\end{array}$ & 134,135 \\
\hline H.i. & Fruits aqueous extract & in vivo - hypoglycemia in patients with type II diabetes & 22 \\
\hline H.i. & $n$-butanolic extract & in vivo - regulates blood glucose levels & 136 \\
\hline H.i. & Fruits ethanolic extract & in vivo - regulates blood glucose levels & 137 \\
\hline
\end{tabular}


Table 3. In vitro, in vivo, ex vivo and in silico biological studies reported from Helicteres genus (cont.)

\begin{tabular}{|c|c|c|c|}
\hline Species & Material used & Experimental model & Literature \\
\hline H.a. & Roots aqueous extract & in vivo - increases glucose uptake & 138 \\
\hline H.a. & Roots ethanolic extract & in vivo - increases the uptake of glucose and adipocytes & 139 \\
\hline H.a. & - & in vivo - inhibition of $\alpha$-glicosidase & 138,139 \\
\hline \multicolumn{4}{|c|}{ Antioxidant Activity } \\
\hline H.i. & Bark aqueous extract & in vitro - inhibition of peroxidation radicals & 81 \\
\hline H.i. & Fruit hot aqueous extract & in vitro - inhibition of radicals $\mathrm{H}_{2} \mathrm{O}_{2}$ and $\mathrm{NO}$ & 27 \\
\hline H.i. & Fruits phenolic extracts & in vitro - inhibition of radicals ABTS, Hydroxyl and DPPH & 140 \\
\hline H.i. & Fruits aqueous extract & in vitro - inhibition of radicals DPPH and TBARS & 129 \\
\hline H.i. & Fruits aqueous extract & in vitro - inhibition of radicals Hydroxyl, $\mathrm{H}_{2} \mathrm{O}_{2}$ and DPPH & 141 \\
\hline H.i. & Fruits extract & in vitro - inhibition of radicals DPPH & 107 \\
\hline H.i. & Fruits extract & in vitro - inhibition of radicals FRAP and DPPH & 142,143 \\
\hline H.i. & Fruits extract & in vitro - inhibition of radicals DPPH and $\mathrm{H}_{2} \mathrm{O}_{2}$ & 107 \\
\hline H.i. & Fruits and barks extracts & in vitro - inhibition of lipid peroxidation & 142 \\
\hline H.i. & Roots aqueous extract & in vitro - inhibition of radicals $\mathrm{ABTS}$ and $\mathrm{DPPH}$ & 144 \\
\hline H.i. & Leaves, barks, roots and fruits extracts & in vitro - inhibition of radicals FRAP and DPPH & 145 \\
\hline H.i. & Leaves and fruits extracts & in vitro - inhibition of radical DPPH & 146 \\
\hline H.a. & Polysaccharides & in vitro - inhibition of radicals ABTS, Hydroxyl and DPPH & $147-149$ \\
\hline H.a. & Roots ethanolic and aqueous fractions & in vitro - inhibition of $\mathrm{ABTS}$ and $\mathrm{DPPH}$ & 150 \\
\hline H.h. & Leaves extracts & in vitro - inhibition of radicals ABTS, DPPH and FRAP & 148 \\
\hline H.h. & Fraction rich in saponin & in vitro - inhibition of radicals ABTS, DPPH, FRAP and CUPRAC & $\begin{array}{c}24,45,95,111 \\
151\end{array}$ \\
\hline H.h. & Stem and Leaves extracts & in vitro - inhibition of radicals, ABTS, DPPH and FRAP & $45,95,96$ \\
\hline H.veg. & Stem extracts & in vitro - inhibition of radicals, $\mathrm{ABTS}$ and $\mathrm{DPPH}$ & 47 \\
\hline \multicolumn{4}{|c|}{ Antimicrobial and Antiviral Activity } \\
\hline$\overline{\text { H.i. }}$ & Fruits extracts & $\begin{array}{c}\text { in vitro - E. coli, Staphylococcus epidermidis, Salmonella typhimurium } \\
\text { and Proteus vulgaris }\end{array}$ & 152 \\
\hline H.i. & Fruits acetone extracts & in vitro - Enterococcus faecalis, Escherichia coli and Bacillus cereus & 153 \\
\hline H.i. & Leaves ethanolic extract & in vitro - Escherichia coli, Steptococcus and Salmonella & 154 \\
\hline H.i. & Leaves and fruits extracts & $\begin{array}{c}\text { in vitro - Staphylococcus aureus, Bacillus subtilis and B. coagulans; } \\
\text { Escherichia coli, Pseudomonas aeruginosa and Salmonella typhi; } \\
\text { Bipolaris sorokiniana, Fusarium oxysporum f.sp. zingiberi, Colletotri- } \\
\text { chum capsici and Curvularia sp. }\end{array}$ & 150 \\
\hline H.i. & Stem and leaves extracts & $\begin{array}{c}\text { in vitro - E. coli, Pseudomonas, S. aureus, Bacillus subtillis, Aspergil- } \\
\text { lus fumigatous, Aspergillus awamori, Rhizopus oryzae, Tricoderma } \\
\text { viridae and Culmularia oryzae }\end{array}$ & 155 \\
\hline H.i. & Fruits aqueous extract & in vitro - Pseudomonas aeruginosa & 156 \\
\hline H.i. & Oleanolic acid & in vitro - S. aureus, E coli, $P$. aeruginosa, B. cereus and A. flavus & 88 \\
\hline H.i. & $\beta$-sitosterol & $\begin{array}{c}\text { in vitro - E. coli, Pseudomonas aeruginosa, Staphylococcus aureus and } \\
\text { Bacillus cereus }\end{array}$ & 88 \\
\hline H.i. & Barks and stems extracts & $\begin{array}{c}\text { in vitro - Cryptococcus neoformans, Candida tropicalis, Trychophyton } \\
\text { rubrum, Microsporum furfure and Epidermophyton floccosum }\end{array}$ & 157 \\
\hline H.i. & Roots hydroethanolic extract & $\begin{array}{c}\text { in vitro - Bacillus subtilis; Micrococcus luteus; } S . \text { aureus; E. coli; } P . \\
\text { vulgaris; P. aeruginosa; S. typhimurium; A. niger; C. albicans and } S . \\
\text { cerevisiae }\end{array}$ & 101 \\
\hline H.i. & Fruits, barks and leaves extracts & $\begin{array}{c}\text { in vitro - Escherichia coli, Salmonella typhii, Staphylococcus aureus, } \\
\text { Corynebacteria diphtheriae and Nocardia } \mathrm{sp} .\end{array}$ & 158 \\
\hline H.i. & Leaves ethanolic extract & $\begin{array}{c}\text { in vitro - Pseudomonas aeruginosa, Staphylococcus aureus and Asper- } \\
\text { gillus niger }\end{array}$ & 159 \\
\hline H.i. & Fruits methanolic extract & in vitro - Candida albicans & 148 \\
\hline H.i. & $\begin{array}{l}\text { 10-methyl, 4-isopropenyl, dodecahydro- ethanophenan- } \\
\text { threne }\end{array}$ & not reported & 65 \\
\hline H.a. & not reported & in vitro - E. coli & 160 \\
\hline H.a. & Triterpenes & in vivo and in vitro - Hepatitis B & 161 \\
\hline H.a. & Methyl helicterate & in vivo and in vitro - anti $\mathrm{HBV}$ & 162,163 \\
\hline H.h. & Roots extract & in vitro - Staphylococcus aureus and Lactobacillus fermentum & 114 \\
\hline H.h. & Saponin-enriched fractions & in vitro - E. coli and S. lugdunensis & 164 \\
\hline H.gr & Aerial parts extract & $\begin{array}{l}\text { in vitro - Bacillus subtilis; Micrococcus luteus; Enterobacter cloacae; } \\
\text { Acinetobacter calcoaceticus; Aspergilus oryzae; Curvularia luneta; } \\
\text { Mucor } \mathrm{sp.}\end{array}$ & 165 \\
\hline
\end{tabular}


Table 3. In vitro, in vivo, ex vivo and in silico biological studies reported from Helicteres genus (cont.)

\begin{tabular}{|c|c|c|c|}
\hline Species & Material used & Experimental model & Literature \\
\hline \multicolumn{4}{|c|}{ Other Activities } \\
\hline H.i. & Fruits extract & in vitro and in vivo - antispasmodic & 37 \\
\hline H.i. & Fruits extract & in vitro - cardiotonics & 166 \\
\hline H.i. & Fruits aqueous extract & in vivo - anthelminthic & 167 \\
\hline H.i. & Fruits extract & in vivo - anthelminthic & 144 \\
\hline H.i. & Bark methanolic extract & in vitro - cytoprotectors & 168 \\
\hline H.i. & Bark aqueous extract & in vivo - acute oral toxicity & 39 \\
\hline H.i. & $\begin{array}{l}\text { 10-methyl, 4-isopropenyl, dodecahydro- ethanophenan- } \\
\text { threne }\end{array}$ & in vivo - antispasmodic & 65 \\
\hline H.i. & Fruit extract & Demulcent and Astringent & 169 \\
\hline H.a. & Terpenoids & in silico - ulcerative cults & 170,171 \\
\hline H.a. & Leaves hydroethanolic extract & in vivo - acute and subchronic toxicity & 8 \\
\hline H.vel. & Aerial parts extract & in vitro - Larvicidal activity against Aedes aegypti & 12 \\
\hline H.vel. & Tiliroside and 7,4'-di- $O$-methyl-8- $O$-sulphate flavone & in silico and in vitro - Larvicidal activity against Aedes aegypti & 89 \\
\hline H.s. & Hydroethanolic extract & in vivo - gastroprotective & 8 \\
\hline H.s. & Hydroethanolic extract & in vivo - acute and subchronic oral toxicity & 8 \\
\hline H.e. & Aerial parts extract & in vitro - Larvicidal activity against Aedes aegypti & 48 \\
\hline H.veg & Leaves and stem extracts & in vitro - Artemia salina eggs & 47 \\
\hline
\end{tabular}

H.i.: H. isora; H.a.: H. angustifolia; H.vel.: H. velutina; H.h.: H. hirsuta; H.veg.: H. vegae; H.s.: H. sacarolha; H.ga.: H. gardineriana; H.e.: H. eichleri; H.gr: H. grazumifolia.

The acetone extract of $H$. isora fruits exhibited better cytotoxic activity in vitro against lung cancer cells. ${ }^{107}$ Studies with the terpenes Helicteric acid (38), oleanolic acid (45) and betulinic acid (12) isolated from $H$. angustifolia have shown important anticancer activity and showed that compounds could decrease proliferation and induce apoptosis in HT-29 colorectal cancer cells in vitro.$^{60} \mathrm{~A}$ similar activity was developed by the compounds $2,3,3 \beta-O-[(E)$-coumaroyl $]$ betulinic acid (15) and pyracrenic acid (16). ${ }^{44}$

In vivo studies revealed that hydroethanolic extract flavonoidrich of $H$. sacarolha and phenolic compounds have good activity in ovarian cancer cell lineages, being non-toxic when ingested orally, ${ }^{8}$ while hidroethanolic stem bark extract of $H$. isora shows activity against hepatocellular carcinoma in mice. ${ }^{167}$

\section{Hepatoprotective activity}

Extracts of several plant species have shown hepatoprotective activity ${ }^{176-178}$ and approximately 100 of these species have been used in the preparation of over 700 herbal formulations that are available for use in prevention and treatment of liver disease. ${ }^{179,180}$

The hepatic protection exerted by the H. isora and H. angustifolia species was also investigated in vivo, where the main parameters of alterations in liver enzymes production and serum markers are evaluated. $H$. isora bark ethanolic extract and $H$. angustifolia water extract demonstrated hepatoprotective activity against carbon tetrachloride induced liver damage in rats and mice, respectively. ${ }^{116}$ The methyl helicterate (30) isolated from $H$. angustifolia acts on carbon tetrachloride in induced hepatic fibrosis of rats, which may be associated with its free radical scavenging action and antioxidant activity. Another proposed mechanism of action of this substance would be the inhibits activation of hepatic stellate cells, modulating apoptosis and autophagy. ${ }^{120,121}$

\section{Antidiabetic and hypolipidemic activity}

Available literature shows that various chemical compounds with antidiabetic properties have been identified in some plant species, ${ }^{181,182}$ among which we can highlight some belonging to the Helicteres genus.

The ethanolic extract of $H$. isora roots causes significant reduction in glucose, triglyceride and insulin levels in mouse plasma, suggesting that it has insulin sensitizing and hypolipidemic activity with potential use in the treatment of type 2 diabetes. ${ }^{28}$ Researches over this species have also proven the antidiabetic activity of the aqueous extracts of its peels, stem and fruits. ${ }^{30,127,129}$

The extract and n-butanolic fractions of $H$. isora have shown good in vivo activity with antihyperglycemic activity, reducing glucose and total cholesterol levels. ${ }^{123,124}$ Saponin-rich fractions also exhibit this activity in vitro and in vivo. ${ }^{126,130}$ Molecular docking with insulin receptors was analyzed with compounds isolated from $H$. isora fruits, ${ }^{183}$ and the results suggested that they may be useful for treating diabetes.

$H$. angustifolia roots aqueous and ethanolic extracts have also shown significant antidiabetic activity in vivo, significant alphaglucosidase inhibitory and moderate enhanced glucose consumption activity, while having low cytotoxicity and acute toxicity. ${ }^{138,139}$

\section{Antioxidant activity}

Antioxidants are important for preventing human diseases. Naturally occurring antioxidants such as ascorbic acid, vitamin E and phenolic compounds can reduce the oxidative damage associated with various diseases including cancer, cardiovascular disease, cataract, atherosclerosis, diabetes, arthritis, immune deficiency diseases and aging. ${ }^{27}$

Evaluation of antioxidant activity of the species $H$. isora, $H$. angustifolia, $H$. hirsuta and $H$. vegae, mainly with respect to fruit extract, ${ }^{141}$ rich fractions of saponins ${ }^{24}$ and polissacarids, ${ }^{147}$ showed that they are capable of inhibiting in vitro peroxidation radicals such as DPPH (1,1-diphenyl-2-picryl-hydrazyl), $\mathrm{H}_{2} \mathrm{O}_{2}$ (Hydrogen peroxide), NO (Nitric Oxide), ABTS (2,2'-azino-bis(3-ethylbenzothiazoline-6sulphonic acid)), TBARS (thiobarbituric acid-reactive substances), FRAP (ferric reducing antioxidant power) and CUPRAC (cupric reducing antioxidant capacity).

\section{Antimicrobial and antiviral activity}

In the current scenario, due to the various pathogenic microorganisms, infectious diseases are still one of the leading reasons behind the worldwide death rates. ${ }^{184}$ The emergence of 
multiple commonly used antibiotic drug resistant bacteria is a severe health problem and major challenge for global drug discovery programs, ${ }^{154}$ and the use of plant extracts and isolated compounds with known antimicrobial properties becomes an important alternative in therapeutic treatments. ${ }^{185}$

$H$. isora and $H$. angustifolia organic extracts have been extensively studied for their potential to act as antimicrobial agents. ${ }^{150}$ Among the isolated compounds, oleanolic acid $(\mathbf{4 5})^{88}$ and $\beta$-sitosterol $(\mathbf{5 1})^{88}$ showed good antibacterial activity, while methyl helicterilate compound (49) showed potential antiviral activity ${ }^{162}$ (Table 3).

\section{Other activities}

Other activities have been reported from Helicteres species. $H$. isora stems aqueous extract showed no toxicity when administered orally at concentrations of 100 and $200 \mathrm{mg} / \mathrm{kg}$ in rats. ${ }^{39}$ Researchers also evaluate antispasmodic, ${ }^{37}$ gastroprotective, ${ }^{8}$ anthelmintic ${ }^{167}$ and larvicide against Aedes aegypti larvae ${ }^{12,48}$ activities, among others. In addition, studies were also carried out to evaluate the nutritional value of $H$. isora fruits and stems. ${ }^{186}$

\section{CONCLUSIONS}

Helicteres L. is one of the genera belonging to Sterculiaceae clade in Malvaceae family with several notable activities. Previous studies have revealed that terpenoids, flavonoids and lignoids are the dominant constituents of Helicteres species. However, information about this genus is scarce and not systematic. The in vitro and in vivo studies carried out to date prove traditional medicine reports regarding the activities of those species. However, pharmacological tests with isolated substances are still rare from this genus and its compounds, especially those unpublished in the literature, resulting in unexplored potentials.

Given the presented data, it is extremely important to continue exploring the chemical and biological potentials of these and other species present in the American and Asian flora, considering the need to find substances with biological activities that may be used for mankind benefit.

\section{ACKNOWLEDGMENTS}

The authors thank the Coordenação de Aperfeiçoamento do Ensino Superior (CAPES) and the Conselho Nacional de Desenvolvimento Científico e Tecnológico (CNPq).

\section{REFERENCES}

1. Eloy, C. C.; Vieira, D. M.; Lucena, C. M.; Andrade, M. O.; Gaia Scientia 2014, Volume Especial Populações Tradicionais, 189.

2. Machado, V. R.; Lang, K. L.; Durán, F. J.; Cabrera, G. M.; Palermo, J. A.; Schenkel, E. P.; Bernardes, L. S. C.; Quim. Nova 2015, 38, 37.

3. Barreiro, E. J.; Bolzani, V. S.; Quim. Nova 2009, 32, 679.

4. Oliveira, L. F. G.; Gilbert, B.; Villas-Bôas, G. K.; Rev. Fitos 2013, 8, 33.

5. Bonomi, T. J.; Góes, J. A.; Machado, M. S.; Silva, R. M. L.; Malheiros, A.; Quim. Nova 2018, 41, 36.

6. Deshpande, H. A.; Bhalsing, S. R.; Res. Biotechnol. 2015, 6, 31.

7. Teles, Y. C. F.; Gomes, R. A.; Oliveira, M. S.; Lucena, K. L.; Nascimento, J. S.; Agra, M. F.; Igoli, J. O.; Gray, A. I.; Souza, M. F. V.; Quim. Nova 2014, 37, 1491.

8. Balogun, S. O.; Damazo, A. S.; Martins, D. T. O.; J. Ethnopharmacol. 2015, 166, 176.

9. Ya, T.; Gilbert, M. G.; Dorr, L. J.; Flora of China 2007, 12, 240.

10. Cruz, F. R.; Master's thesis, Universidade de São Paulo, Brasil, 2007.

11. Goldberg, L.; Rev. Biol. Trop. 2009, 57, 161.
12. Fernandes, D. A.; Souza, M. S. R.; Teles, Y. C. F.; Oliveira, L. H. G.; Lima, J. B.; Conceição, A. S.; Nunes, F. C.; Silva, T. M. S.; Souza, M. F. V.; Molecules 2018, 23, 2784.

13. Cristóbal, C. L.; Bonplandia 2001, 11, 1 .

14. Silva, C. A.; Ferreira, D. S.; Koch, A. K.; Araújo-Silva, L. E.; Acta. Bot. Bras. 2010, 24, 462.

15. https://www.flickr.com/photos/tags/helicteres, accessed in September 2019.

16. Sazima, M.; Plant Biol. 1988, 101, 269.

17. Franceschinelli, E. V.; Kesseli, R.; Heredity 1999, 82, 355.

18. Franceschinelli, E. V.; Bawa, K. S.; Heredity 2000, 84, 116.

19. Franceschinelli, E. V.; Bawa, K. S.; Braz. J. Bot. 2005, 28, 163.

20. González, A. M.; Cristóbal, C. L.; Bonplandia 1997, 287.

21. Atluri, J. B.; Rao, S. P.; Reddi, C. S.; Curr. Sci. 2000, 78, 713.

22. Venkatesh, S.; Reddy, G. D.; Reddy, Y. S. R.; Sathyavathy, D.; Reddy, B. M.; Fitoterapia 2004, 75, 364

23. Purnomo, L.; Darsono, L.; Santosa, S.; Journal Kedokteran Maranatha 2010, 3, 39 .

24. Dayal, R.; Singh, A.; Ojha, R. P.; Mishra, K. P.; Journal of Medicinal Plants Studies 2015, 3, 95.

25. Li, K.; Lei, Z.; Hu, X.; Sun, S.; Li, S.; Zhang, Z.; J. Ethnopharmacol. 2015, 172,61 .

26. Santos, E. A.; Carvalho, C. M.; Costa, A. L. S.; Conceição, A. S.; Moura, F. B. P.; Santana, A. E. G.; Evidence-Based Complementary Altern. Med. 2012, ID 846583 .

27. Basniwal, P. K.; Suthar, M.; Rathore, G. S.; Gupta, R.; Kumar, V.; Pareek, A.; Jain, D.; Indian J. Nat. Prod. Resour. 2009, 8, 483.

28. Chakrabarti, R.; Vikramadithyan, R. K.; Mullangi, R.; Sharma, V. M.; Jagadheshan, H.; Rao, Y. N.; Sairam, P.; Rajagopalan, R.; J. Ethnopharmacol. 2002, 81, 343.

29. Cunningham, A. B.; Ingram, W.; Brinckmann, J. A.; Nesbitt, M.; J. Ethnopharmacol. 2018, 255, 128.

30. Kumar, G.; Banu, G. S.; Murugesan, A. G.; Pandian, M. R.; J. Ethnopharmacol. 2006, 107, 304.

31. Kumar, G.; Murugesan, A. G.; J. Ethnopharmacol. 2008, 116, 161.

32. Kumar, G.; Banu, G. S.; Murugesan, A. G.; J. Appl. Biomed. 2008, 6, 89.

33. Suthar, M.; Rathore, G. S.; Pareek, A.; Indian J. Pharm. Sci. 2009, 71, 695.

34. Venkatesh, S.; Reddy, B. M.; Reddy, G. D.; Mullangi, R.; Lakshman, M.; J. Nat. Med. 2010, 64, 295 .

35. Chang, Y. S.; Ku, Y. R.; Lin, J. H.; Lu, K. L.; Ho, L. K.; J. Pharm. Biomed. Anal. 2001, 26, 849.

36. Kamiya, K.; Saiki, Y.; Hama, T.; Fujimoto, Y.; Endang, H.; Umar, M.; Satake, T.; Phytochemistry 2001, 57, 297.

37. Pohocha, N.; Grampurohit, N. D.; Phytother. Res. 2001, 15, 49.

38. Satake, T.; Kamiya, K.; Saiki, Y.; Hama, T.; Fujimoto, Y.; Kitanaka, S.; Kimura, Y.; Uzawa, J.; Endang, H.; Umar, M.; Chem. Pharm. Bull. 1999, 47, 1444.

39. Kumar, G.; Banu, G. S.; Murugesan, A. G.; Pandian, M. R.; Iran. J. Pharm. Res. 2007, 6, 123.

40. Chen, C. M.; Chen, Z. T.; Hong, Y. L.; Phytochemistry 1990, $29,980$.

41. Chiu, N. Y.; Chang, K. S.; The Illustrated Medicinal Plants of Taiwan (I); Southern Materials Center, Inc.: Taipei, 1995, p. 104.

42. Wang, M.; Liu, W.; Phytochemistry 1987, 26, 578.

43. Joy, J.; Varanasi, S. B.; Mathew, P. L.; Thomas, S.; Pilla, S.; J. Renewable Mater. 2016, 4, 351.

44. Pan, M. H.; Chen, C. M.; Lee, S. W.; Chen, Z. T.; Chem. Biodiversity 2008, 5, 565 .

45. Pham, H. N. T.; Nguyen, V. T.; Vuong, Q. V.; Bowyer, M. C.; Scarlett, C. J.; J. Food Process. Preserv. 2017, 41, 1745.

46. Chin, Y. W.; Jones, W. P.; Rachman, I.; Riswan, S.; Kardono, L. B. S.; Chai, H. B.; Farnsworth, N. R.; Cordell, G. A.; Swanson, S. M.; Cassady, J. M.; Kinghorn, A. D.; Phytother. Res. 2006, 20, 62 
47. Olivas-Quintero, S.; López-Ângulo, G.; Montes-Avila, J.; DíazCamacho, S. P.; Vega-Aviña, R.; López-Valenzuela, J. Á.; Salazar-Salas, N. Y.; Delgado-Vargas, F.; Pharm. Biol. 2017, 55, 1473.

48. Assis, E. B.; Master's thesis, Universidade Federal da Paraíba, Brasil, 2019.

49. Bean, M. F.; Master's thesis, Purdue University, West Lafayette, USA, 1982.

50. Chen, Z. T.; Lee, S. W.; Chen, C. M.; Chem. Pharm. Bull. 2006, 54, 1605.

51. Chen, W.; Tang, W.; Lou, L.; Zhao, W.; Phytochemistry 2006, 67, 1041.

52. Wang, G. C.; Li, T.; Wei, Y. R.; Zhang, Y. B.; Li, Y. L.; Sze, S. C. W.; Ye, W. C.; Fitoterapia 2012, 83, 1643.

53. Wei, Y.; Wang, G.; Zhang, X.; Wang, Y.; Ye, W.; China J. Chin. Mater. Med. 2011, 36, 1193.

54. Quang, D. N.; Pham, C. T.; Le, L. T. K.; Ta, Q. N.; Dang, N. K.; Hoang, N. T.; Pham, D. H.; Nat. Prod. Res. 2018, 1478.

55. Qu, W.; Li, J.; Wang, M.; Zhongguo Yaoke Daxue Xuebao 1991, 22, 203.

56. Nguyen, T. T.; Kretschmer, N.; Pferschy-Wenzig, E. M.; Kunert, O.; Bauer, R.; Nat. Prod. Commun. 2019, 14, 7.

57. Yin, X.; Lu, Y.; Cheng, Z. H.; Chen, D. F.; Molecules 2016, 21, 1506.

58. Guo, X.-D.; An, L.-K.; Xu, D.; Ma, L.; Gu, L.-Q.; Chem. J. Chin. Univ. 2003, 24, 2022.

59. Harde, P. A.; Shah, D. R.; Suhagia, B. N.; Shah, M. B.; J. Planar Chromatogr--Mod. TLC 2011, 24, 503.

60. Su, D.; Gao, Y.-Q.; Dai, W.-B.; Hu, Y.; Wu, Y.-F.; Mei, Q.-X.; EvidenceBased Complementary Altern. Med. 2017, ID 5180707.

61. Liu, W. G.; Wang, M. S.; Acta Pharm. Sin. B 1985, 11, 007.

62. Pagi, K. B.; Lahiri, S. K.; Yadav, G. K.; Shah, M. B.; Int. J. ChemTech Res. 2010, 2, 851.

63. Harde, P. A.; Shah, M. B.; Pharmacogn. J. 2017, 9, 523.

64. Sandhya, P.; Grampurohit, N. D.; Pharmacogn. Mag. 2008, 4, 107.

65. Wang, M. S.; Liu, W. G.; Li, J. R.; Shen, F. L.; Lin, X. Y.; Zheng, Q. T.; Acta Chim. Sin. 1988, 46, 768.

66. Guo, X. D.; An, L. K.; Xi, D.; Ma, L.; Gu, L. Q.; Nat. Sci. Ed. 2003, 42, 52.

67. Singh, S. B.; Singh, A. K.; Thakur, R. S.; Indian J. Pharm. Sci. 1984, 46, 148.

68. Jain, P. S.; Badgujar, V. B.; Patil, R. R.; Haswani, N. G.; Chaudhari, S. G.; J. Pharm. Res. 2009, 2, 1397.

69. Ramesh, P.; Yuvarajan, C. R.; J. Nat. Prod. 1995, 58, 1242.

70. Nguyen, T. T.; Gao, X.; Nikles, S.; Pferschy-Wenzing, E. M.; Kunert, O.; Bauer, R.; Reviews of Clinical Pharmacology and Drug Therapy 2017, 15,48 .

71. Buckingham, J.; Munasinghe, V. R. N.; Dictionary of Flavonoids with CD-ROM; CRC Press: Boca Raton, FL, USA, 2015, T-334, 857.

-72. Chen, Z. T.; Lee, S. W.; Chen, C. M.; Heterocycles 1994, 38, 1399.

73. Tezuka, Y.; Terazono, M.; Kusumoto, T. I.; Kawashima, Y.; Hatanaka, Y.; Kadota, S.; Hattori, M.; Namba, T.; Kikuchi, T.; Tanaka, K.; Supriyatna, S.; Helv. Chim. Acta 1999, 82, 408.

74. Tezuka, Y.; Terazono, M.; Kusumoto, T. I.; Hatanaka, Y.; Kadota, S.; Hattori, M.; Namba, T.; Kikuchi, T.; Tanaka, K.; Supriyatna, S.; Helv. Chim. Acta 2000, 83, 2908.

75. Guo, X. D.; Huang, Z. S.; Bao, Y. D.; An, L. K.; Ma, L.; Gu, L. Q.; Chin. Chem. Lett. 2005, 16, 49.

76. Barik, B. R.; Dey, A. K.; Das, P. C.; Indian J. Chem. 1981, $20 B, 938$.

77. Deshpande, H. A.; Bhalsing, S. R.; Physiol. Mol. Biol. Plants 2014, 20 , 89.

78. Kumar, V.; Desai, D.; Shriram, V.; Nat. Prod. Bioprospect. 2014, 4, 107.

79. Ahmad, Z. A.; Indian Drugs 1987, 24, 404.

80. Aleykutty, N. A.; Akhila, S.; Int. J. Comput. Appl. Technol. 2012, 45, 8.

81. Bai, N. S.; Bull. Res. Inst., Univ. Kerala, Trivandrum, Ser. A 1954, 3, 89.

82. Meena, R.; Joshi, R.; Meena, R.; Patni, V.; Indian J. Pharm. Biol. Res. 2016, 4,1 .
83. Meena, N. S. R.; Patni, M. K. M. V.; J. Pharmacogn. Phytochem. 2017, 6, 1213.

84. Yadav, N.; Yadav, R.; Goyal, A.; Int. J. Pharm. Sci. Rev. Res. 2014, 27, 272.

85. Wang, G.; Tang, W.; Bidigare, R. R. In Natural products: Drug discovery and therapeutic medicine; Zhang, L.; Demain, A., eds.; Humana Press: Totowa, NJ, USA, 2005, p. 197.

86. Jansen, D. J.; Shenvi, R. A.; Future Med. Chem. 2014, 6, 1127.

87. Santos, R. A. F.; Master's thesis, Universidade Federal da Bahia, Brazil, 2010.

88. Kumar, D.; Singh, R. K.; Farooq, S.; World J. Pharm. Res. 2017, 6, 1102.

89. Fernandes, D. A.; Barros, R. P. C.; Teles, Y. C. F.; Oliveira, L. H.; Lima, J. B.; Scotti, M. T.; Nunes, F. C.; Conceição, A. S.; Souza, M. F. V.; Molecules 2019, 24, 2315.

90. Santos, D. S.; Rodrigues, M. M. F.; Estação Científica 2017, 7, 29.

91. Sobrinho, T. J. S. P.; Gomes, T. L. B.; Cardoso, K. C. M.; Amorim, E. L. C.; Quim. Nova 2010, 33, 288.

92. Flambó, D. F. A. L. P.; Master's thesis, Universidade Fernando Pessoa, Porto, Portugal, 2013.

93. Teles, Y. C.; Ribeiro-Filho, J.; Agra, M. F.; Siheri, W.; Igoli, J. O.; Gray, A. I.; Souza, M. F.; Nat. Prod. Res. 2015, 30, 1880.

94. Cao, W.; Hu, C.; Wu, L.; Xu, L.; Jiang, W.; J. Pharm. Sci. 2016, 132, 131.

95. Pham, H. N. T.; Vuong, Q. V.; Bowyer, M. C.; Scarlett, C. J.; Chem. Pap. 2017, 71, 2233.

96. Pham, H. N. T.; Vuong, Q. V.; Bowyer, M. C.; Scarlett, C. J.; Asia-Pac. J. Chem. Eng. 2017, 12, 332.

97. Jain, A.; Sinha, P.; Desai, N. S.; Int. J. Pharm. Sci. Res. 2014, 5, 1320.

98. Sharma, S.; Bhargava, S.; Mehta, A.; World J. Pharm. Pharm. Sci. 2017, $6,1471$.

99. Bhat, B. A.; Int. J. Bioassays 2012, 1, 177.

100. Sousa, E. T. S.; Lopes, W. A.; Andrade, J. B.; Quim. Nova 2016, 39, 486.

101. Venkatesh, S.; Laxmi, K. S.; Reddy, B. M.; Ramesh, M.; Fitoterapia 2007, 78, 146

102. He, Y.; Li, Y. P.; Yang, W.; Zeng, X. T.; Xiao, B. Q.; Nie, J. Y.; Strait Pharm. J. 2009, 28.

103. Shukla, N.; Goswami, R. B.; World J. Pharm. Res. 2016, 5, 1420.

104. Jiang, C.; Wu, M.; Qin, Q.; Meng, X.; Liu, B.; China J. Tradit. Chin. Med. Pharm. 2010, 25, 1672.

105. de Melo, J. O.; de Arruda, L. L. M.; Baroni, S.; Truiti, M. C. T.; Caparroz-Assef, S. M.; Cuman, R. K. N.; Bersani-Amado, C. A.; Evidence-Based Complementary Altern. Med. 2012, ID 141947.

106. Melo, J. O.; Pedrochi, F.; Baesso, M. L.; Hernandes, L.; Truiti, M. C. T.; Baroni, S.; Bersani-Amado, C. A.; Pharm. Res. 2011, 28, 331.

107. Raaman, N.; Balasubramanian, K.; J. Acad. Ind. Res. 2012, 1, 148.

108. Dayal, R.; Singh, A.; Mishra, K. P.; J. Pharmacogn. Phytochem. 2017, $6,417$.

109. Bean, M. F.; Antoun, M.; Abramson, D.; Chang, C. J.; McLaughlin, J. L.; Cassady, J. M.; J. Nat. Prod. 1985, 48, 500.

110. Sharma, V.; Shori, A.; Paliwal, S. K.; Sharma, S.; Asian J. Pharm. Clin. Res. 2015, 8, 344

111. Li, K.; Yu, Y.; Sun, S.; Liu, Y.; Garg, S.; Kaul, S. C.; Lei, Z.; Gao, R.; Wadhwa, R.; Zhang, Z.; PLoS One 2016, 11, e0152017.

112. Sun, S.; Li, K.; Lei, Z.; Xiao, L.; Gao, R.; Zhang, Z.; Biomed. Pharmacother. 2018, 101, 881.

113. Li, K. J.; Yang, X.; Hu, X. S.; Han, C.; Lei, Z. F.; Zhang, Z. Y.; J. Taiwan Inst. Chem. Eng. 2016, 61, 75.

114. Dzung, D. T. T.; Trang, T. H.; Linh, L. T. K.; Tan, D. V.; Hoa, L. T. P.; Tap Chi Sinh Hoc 2018, 40, 45.

115. Kumar, G.; Murugesan, A. G.; Pandian, M. R.; Pharm. Unserer Zeit 2006, 353

116. Kumar, N.; Singh, A. K.; Asian-Pac. J. Trop. Biomed. 2014, 4, S22. 
117. Sahane, R.; Kanade, P.; Nigade, P.; Chaudhari, H.; Int. J. Appl. Res. Nat. Prod. 2017, 3, 169.

118. Dhevi, R.; Gayathri, K.; Shabi, M. M.; Subashini, U.; Dubey, G. P.; Rajamanickam, G. V.; Chitra, M.; Bulg. J. Vet. Med. 2008, 11, 235.

119. Lin, X.; Huang, Q. F.; Zhang, S. J.; Liao, M.; Huang, R. B.; Chin. J. Exp. Tradit. Med. Formulae 2010, 147.

120. Huang, Q.; Li, Y.; Zhang, S.; Huang, R.; Zheng, L.; Wei, L.; He, L.; Liao, M.; Li, L.; Zhuo, L.; Lin, X.; J. Ethnopharmacol. 2012, 143, 889.

121. Zhang, X. L.; Chen, Z. N.; Huang, Q. F.; Bai, F. C.; Nie, J. L.; Lu, S. J.; Wei, J. B.; Lin, X.; Cell. Physiol. Biochem. 2018, 51, 897.

122. Raja, A. B.; Elanchezhiyan, C.; Sethupathy, S.; Riv. Eur. Sci. Med. Farmacol. 2010, 14, 191.

123. Venkatesh, S.; Reddy, G. D.; Reddy, B. M.; Pharm. Biol. 2003, 41, 347.

124. Tiwari, V.; Singh, A.; Tiwari, A.; Nat. Prod. J. 2012, 2, 9.

125. Kanthale, P. R.; Biradar, S.; Pharmaceutical and Biological Evaluations 2017, 4, 47.

126. Bhavsar, S. K.; Singh, S.; Giri, S.; Jain, M. R.; Santani, D. D.; J. Ethnopharmacol. 2009, 124, 426.

127. Kumar, G.; Banu, G. S.; Murugesan, A. G.; Hum. Exp. Toxicol. 2009, $28,689$.

128. Kumar, G.; Banu, G. S.; Murugesan, A. G.; Food Chem. Toxicol. 2009, 47, 1803.

129. Vijay, K. P.; Laxman, B. C.; Ashok, R. S.; Bansilal, S. S.; Janardhan, P. M.; J. Biol. Sci. Opin. 2013, 1, 5.

130. Bhavsar, S. K.; Foller, M.; Gu, S. C.; Vir, S.; Shah, M. B.; Bhutani, K. K.; Santani, D. D.; Lang, F.; J. Ethnopharmacol. 2009, 126, 386.

131. Gupta, R. N.; Pareek, A.; Suthar, M.; Rathore, G. S.; Basniwal, P. K.; Jain, D.; Int. J. Diabetes Dev. Countries 2009, 29, 170.

132. Elanchezhiyan, C.; Kumaravel, K.; Bhat, B. A.; Sethupathy, S.; Pharmacogn. J. 2014, 6, 86.

133. Elanchezhiyan, C.; Kumaravel, K.; Bhat, B. A.; Sethupathy, S.; J. Pharm. Res. 2014, 8, 243.

134. Alok, S.; Vijayakumar, M.; Raj, V. A.; Md, T.; Unnikrishnan, M. K.; Int. J. Pharm. Clin. Res. 2009, 1, 15.

135. Soma, V.; Zareen, N.; Rajkumari, P.; Reddy, M.; Clin. Immunol. 2008, 127, S104.

136. Vennila, S.; Bupesh, G.; Dhanakaran, D.; Magesh, S.; Senthilkumar, V.; Senthilraja, R.; Saravanamurali, K.; Saran, N.; J. Diabetes Metab. 2015, $6,473$.

137. Yadav, B. V.; Bodhankar, S. L.; Dhaneshwar, S. R.; Pharmacologyonline 2008, 3, 820.

138. Hu, X.; Cheng, D.; Zhang, Z.; Pharm. Biol. 2016, 54, 938.

139. Hu, X. S.; Cheng, D. L.; Li, K. J.; Wang, L. B.; Yang, X.; Sun, S.; Wang, Y. P.; Li, S. H.; Lei, Z. F.; Zhang, Z. Y.; Riv. Eur. Sci. Med. Farmacol. 2016, 20, 1423 .

140. Loganayaki, N.; Siddhuraju, P.; Manian, S.; J. Food Sci. Technol. 2013, $50,687$.

141. Kumar, V.; Sharma, M.; Lemos, M.; Shriram, V.; J. Pharm. Res. 2013, 6,620 .

142. Babu, P. B. R.; Krishnamoorthy, P.; Deepthi, N.; Nissi, M.; J. Drug Delivery Ther. 2013, 3, 33.

143. Rattanamaneerusmee, A.; Thirapanmethee, K.; Nakamura, Y.; Bongcheewin, B.; Chomnawang, M. T.; Res. Pharm. Sci. 2018, 13, 484.

144. Manke, M. B.; Dhawale, S. C.; Patil, D. A.; Pekamwar, S. S.; Jamkhande, P. G.; J. Biol. Act. Prod. Nat. 2015, 5, 18.

145. Devi, S. G.; Sivagami, S.; Saroja, S.; Plant Arch. 2010, 10, 733.

146. Chaudhary, U.; Sharma, V.; Sharma, S.; World J. Pharm. Res. 2016, 5, 1352.

147. Jain, A.; Ranade, R.; Pritam, P.; Joshi, N.; Vavilala, S. L.; Jain, A.; Am. J. Life Sci. 2014, 2, 292.

148. Liu, Q.; Ge, X.; Chen, L.; Cheng, D.; Yun, Z.; Xu, W.; Shao, R.; Int. J. Biol. Macromol. 2018, 107, 2262.
149. Sun, S.; Li, K.; Xiao, L.; Lei, Z.; Zhang, Z.; Biomed. Pharmacother. 2019, 109, 262

150. Akshatha, S.; Chaithra, K. J.; Priyanka, A.; Priyanka, L.; Prashith, K. T. R.; Raghavendra, H. L.; World J. Pharm. Pharm. Sci. 2015, 4, 1793.

151. Pham, H. N. T.; Nguyen, V. T.; Vuong, Q. V.; Bowyer, M. C.; Scarlett, C. J.; Technologies 2015, 3, 285.

152. Tambekar, D. H.; Khante, B. S.; Panzade, B. K.; Dahikar, S. B.; Banginwar, Y. S.; Afr. J. Tradit., Complementary Altern. Med. 2008, 5 , 290.

153. Shriram, V.; Jahagirdar, S.; Latha, C.; Kumar, V.; Dhakephalkar, P.; Rojatkar, S.; Shitole, M. G.; Indian J. Med. Res. 2010, 132, 94.

154. Anburaja, V.; Nandagopalan, V.; Prabha, A. L.; J. Ecobiol. 2010, 26, 211.

155. Pandey, A.; Upadhyaya, A.; Pande, K. K.; Int. J. Bioassays 2015, 4, 4127.

156. Mapara, N.; Sharma, M.; Shriram, V.; Bharadwaj, R.; Mohite, K. C.; Kumar, V.; Appl. Microbiol. Biotechnol. 2015, 99, 10655.

157. Badgujar, V. B.; Jain, P. S.; Int. J. PharmTech Res. 2009, 1, 1376.

158. Varghese, E.; Narayanan, S. S.; Gopal, R. V.; Nair, A.; Chittethu, A. B.; Anson, T. A.; Int. J. Pharm. Technol. 2011, 3, 2560.

159. Subbalakshmi, C.; Meera, B. G.; Pullaiah, T.; Int. J. Green Herb. Chem. 2015, 4, 92

160. Si, H.; Huang, K.; Zha, W.; Xia, J.; Wu, Y.; Liu, Z.; Zhao, Y.; Sun, Y.; Hu, M.; CN 105726588 A 2016.

161. Lin, X.; He, M.; Huang, Q.; Liao, M.; Huang, R.; Zhang, S.; $C N$ 102188461 A 2011.

162. Huang, Q. F.; Yang, H.; Wei, G.; Lin, X.; Zhang, S. J.; Huang, R. B.; Chin. J. Exp. Tradit. Med. Formulae 2011, 179.

163. Huang, Q.; Huang, R.; Wei, L.; Chen, Y.; Lv, S.; Liang, C.; Zhang, X.; Yin, F.; Li, H.; Zhuo, L.; Lin, X.; Antiviral Res. 2013, 100, 373.

164. Pham, H. N. T.; Sakoff, J. A.; Bond, D. R.; Vuong, Q. V.; Bowyer, M. C.; Scarlett, C. J.; Mol. Biol. Rep. 2018, 45, 2125.

165. Armas, H. D.; Vásquez, V.; Ordaz, G.; Int. J. Herb. Med. 2018, 6, 47.

166. Dama, G. Y.; Tare, H. L.; Gore, M. S.; Shende, V. S.; Deore, S. R.; Khandagale, S. T.; Kandekar, A. E.; Int. J. Preclin. Pharm. Res. 2011, 2,81 .

167. Shah, D. V.; Shyale, S. S.; Soloman, S. S.; World J. Pharm. Pharm. Sci. 2015, 4, 788

168. Pradhan, M.; Sribhuwaneswari, S.; Karthikeyan, D.; Minz, S.; Sure, P.; Chandu, A. N.; Mishra, U.; Kamalakannan, K.; Saravanankumar, A.; Sivakumar, T.; J. Pharm. Technol. 2008, 1, 450.

169. Malarvizhl, M.; Kunthavi, B.; Kandhimathi, A. S. P.; Int. J. Curr. Res. Chem. Pharm. Sci. 2018, 5, 1.

170. Gao, Y. Q.; Su, D.; Hu, Y.; Lin, H.; Zhang, W. X.; Dai, W. B.; J. Chin. Med. Mater. 2013, 36, 597.

171. Gao, Y. Q.; Su, D.; Zhang, Y. Y.; Weng, S. F.; He, Z. H.; Mei, Q. X.; Lishizhen Med. Mater. Med. Res. 2014, 25, 1369.

172. Lalrinzuali, K.; Vabeiryureilai, M.; Jagetia, G. C.; Int. J. Inflammation 2016, ID 8247014.

173. Ior, L. D.; Otimenyin, S. O.; Umar, M.; J. Pharm. 2012, 2, 33

174. Jeena, K.; Liju, V. B.; Kuttan, R.; Int. J. Pharm. Pharm. Sci. 2015, 7, 341.

175. Cardoso, M. F. C.; da Silva, I. M. C. B.; dos Santos Júnior, H. M.; Rocha, D. R.; Araújo, A. J.; Pessoa, C.; de Moraes, M. O.; Lotufo, L. V. C.; da Silva, F. C.; Santos, W. C.; Ferreira, V. F.; J. Braz. Chem. Soc. 2013, 24, 12.

176. Lin, E.-Y.; Chagnaadorj, A.; Huang, S.-J.; Wang, C.-C.; Chiang, Y.-H.; Cheng, C.-W.; Evidence-Based Complementary Altern. Med. 2018, ID 4130307.

177. Yahya, F.; Mamat, S. S.; Kamarolzaman, M. F. F.; Seyedan, A. A.; Jakius, K. F.; Mahmood, N. D.; Shahril, M. S.; Suhaili, Z.; Mohtarrudin, N.; Susanti, D.; Somchit, M. N.; Teh, L. K.; Salleh, M. Z.; Zakaria, Z. A.; Evidence-Based Complementary Altern. Med. 2013, ID 636580

178. Akther, N.; Shawl, A. S.; Sultana, S.; Chadan, B. K.; Akther, M.; J. Pharm. Res. 2013, 7, 565. 
179. Das, K. L. S.; Pharmacogn. Res. 2011, 3, 13.

180. Bhaargavi, V.; Jyotsna, G. S. L.; Tripurana, R.; Int. J. Pharm. Sci. Res. 2014, 5, 690 .

181. Emordi, J. E.; Agbaje, E. O.; Oreagba, I. A.; Iribhogbe, O. I.; BMC Complementary Altern. Med. 2016, 15, 468.

182. Severino, V. G. P.; Monteiro, A. F.; Silva, M. F. G. F.; Lucarini, R.; Martins, C. H. G.; Quim. Nova 2015, 38, 42.
183. Vennila, S.; Bupesh, G.; Saravanamurali, K.; SenthilKumar, V.; SenthilRaja, R.; Saran, N.; Magesh, S.; Bioinformation 2014, 10, 263.

184. Sharma, V.; Chaudhary, U.; Asian J. Pharm. Clin. Res. 2016, 9, 96.

185. Nascimento, G. G. F.; Locatelli, J.; Freitas, P. C.; Silva, G. L.; Braz. J. Microbiol. 2000, 31, 247.

186. Gayathri, P.; Gayathri, D. S.; Srinivasan, S.; Saroja, S.; Hygeia: J. Drugs Med. 2010, 2, 57. 